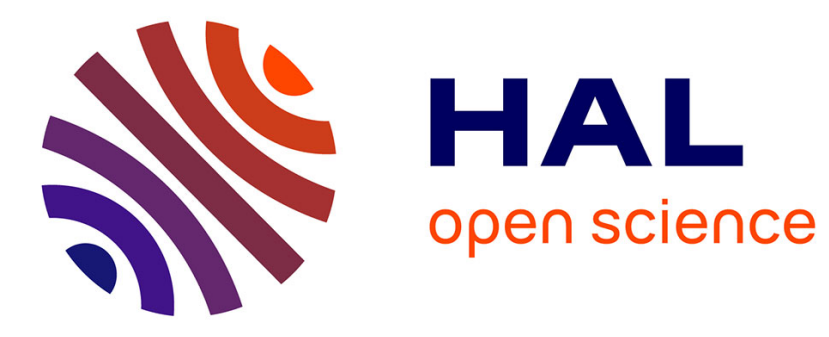

\title{
Semi-parametric estimation for heavy tailed distributions
}

Gabriela Ciuperca, Cécile Mercadier

\section{To cite this version:}

Gabriela Ciuperca, Cécile Mercadier. Semi-parametric estimation for heavy tailed distributions. Extremes, 2010, 13 (1), pp.55-87. 10.1007/s10687-009-0086-6 . hal-00292587

\section{HAL Id: hal-00292587 \\ https://hal.science/hal-00292587}

Submitted on 2 Jul 2008

HAL is a multi-disciplinary open access archive for the deposit and dissemination of scientific research documents, whether they are published or not. The documents may come from teaching and research institutions in France or abroad, or from public or private research centers.
L'archive ouverte pluridisciplinaire HAL, est destinée au dépôt et à la diffusion de documents scientifiques de niveau recherche, publiés ou non, émanant des établissements d'enseignement et de recherche français ou étrangers, des laboratoires publics ou privés. 


\title{
Semi-parametric estimation for heavy tailed distributions
}

\author{
Gabriela Ciuperca Cécile Mercadier*
}

Université de Lyon, Université Lyon 1, CNRS, UMR 5208 Institut Camille Jordan, Bâtiment du Doyen Jean Braconnier, 43, boulevard du 11 novembre 1918, 69622 Villeurbanne Cedex, France

\begin{abstract}
In this paper, we generalize several works in the extreme value theory for the estimation of the extreme value index and the second order parameter. Weak consistency and asymptotic normality are proven under classical assumptions. Some numerical simulations and computations are also performed to illustrate the finite-sample and the limiting behavior of the estimators.
\end{abstract}

Keywords: Extreme value index, second order parameter, Hill estimator, semi-parametric estimation, asymptotic properties

\section{Introduction}

The estimation of the extreme value index has been widely considered in the literature. It allows to do inference in the far tail of a probability distribution like large quantiles and return periods of high levels estimations. Even when restricting to heavy tail underlying distributions, corresponding to positive value of this parameter, the estimating problem remains interesting, but difficult. Indeed, the accuracy of any estimator depends on the number $k$ of top order statistics to be consider. To be precise, small values of $k$ provide high volatility whereas large values of $k$ induce considerable bias. Hence, semi-parametric extensions may be considered for increasing the degree of freedom in the trade-off between variance and bias.

In this paper we study generalizations of some estimators of the extreme value index, denoted by $\gamma$. More precisely, let us introduce a sample $X_{1}, \ldots, X_{n}$ with continuous distribution function $F$. We assume that $F$ belongs to the max-domain of attraction of the Fréchet distribution with parameter $\gamma>0$, which is equivalent to

$$
\lim _{t \rightarrow \infty} \frac{1-F(t x)}{1-F(t)}=x^{-1 / \gamma}, \quad \forall x>0
$$

For some weighted function $g$ and for some positive real $\alpha$, our statistics of interest have the following form

$$
\Gamma_{n, k}(g, \alpha)=\frac{\frac{1}{k} \sum_{i=1}^{k} g\left(\frac{i}{k+1}\right)\left[\log \frac{X_{n-i+1, n}}{X_{n-k, n}}\right]^{\alpha}}{\int_{0}^{1} g(x)\left(\log \left(x^{-1}\right)\right)^{\alpha} d x},
$$

where $X_{1, n} \leq \ldots \leq X_{n, n}$ denote the order statistics of the sample. This estimator is the well-known Hill estimator (1975) when the parameter $\alpha$ and weights are one. The class of the so-called kernel estimators given by Csörgö et al. (1985), as the one studied in Beirlant et al. (1996), corresponds to a specific form of the weighted function with moreover $\alpha$ equal to one. Note also that Gomes and Martins (1999, 2001) and Segers (2001) considered such type of estimators when the weighted function $g$ is identically equal to one and $\alpha$ is some positive real. Note finally that Hüsler et al. (2006) introduced these weighted estimators when $\alpha$ is equal to one. Consequently, the family of statistics $\Gamma_{n, k}(g, \alpha)$ generalizes several approaches already studied in the literature.

As remarked above, the choice of the number $k$ of order statistics used in the estimation of the extreme value index is of primordial importance. We can define an optimal sequence from the asymptotic mean square error point of view. This sequence can be determined when the underlying distribution has a second order expansion involving an extra unknown parameter, namely the second order parameter that is denoted by $\rho$. We refer for instance to the works of Hall (1982), Dekkers and de Haan (1993) and Draisma et al. (1999).

\footnotetext{
*Corresponding author. Tel.: (0033)4.72.43.16.93; fax: (0033)4.72.43.16.87.

E-mail address: mercadier@math.univ-lyon1.fr
} 
Consequently several estimators of $\rho$ have been studied in articles on the adaptive choice of $k$. See, e.g., Hall and Welsh (1985), Drees and Kaufmann (1998), Gomes et al. (2002) and references therein.

In this paper we also propose three methods in order to estimate the parameter $\rho$. The idea consists in composing a consistent estimator of a function of $\rho$ from differences and quotients of several estimators $\Gamma_{n, k}(g, \alpha)$. Firstly, we consider

$$
\frac{\frac{\Gamma_{n, k}\left(g_{1}, 2 \alpha\right)-\Gamma_{n, k}\left(g_{3}, 1\right)^{2 \alpha}}{\Gamma_{n, k}\left(g_{2}, 2 \ell\right)-\Gamma_{n, k}\left(g_{4}, 1\right)^{2 \ell}}}{\left(\frac{\Gamma_{n, k}\left(h_{1}, \alpha+\ell\right)-\Gamma_{n, k}\left(h_{3}, 1\right)^{\alpha+\ell}}{\Gamma_{n, k}\left(h_{2}, 2 \ell\right)-\Gamma_{n, k}\left(h_{4}, 1\right)^{2 \ell}}\right)^{2}}
$$

for $\alpha$ and $\ell$ some positive reals. Note that this definition extends the class of estimators of Gomes et al. (2002) studied for constant weighted functions and for $\ell$ equal to one. Under some assumptions, we can show that this family of estimators converges in probability. Since the limit does not depend on the parameter $\gamma$, it furnishes a consistent family of estimators of some function of $\rho$. Similarly, we study

$$
\frac{\frac{\Gamma_{n, k}\left(g_{1}, \alpha_{g}\right)-\Gamma_{n, k}\left(g_{3}, 1\right)^{\alpha_{g}}}{\Gamma_{n, k}\left(g_{2}, \alpha_{g}+\ell\right)-\Gamma_{n, k}\left(g_{4}, 1\right)^{\alpha_{g}+\ell}}}{\frac{\Gamma_{n, k}\left(h_{1}, \alpha_{h}\right)-\Gamma_{n, k}\left(h_{3}, 1\right)^{\alpha_{h}+\ell}}{\Gamma_{n, k}\left(h_{2}, \alpha_{h}+\ell\right)-\Gamma_{n, k}\left(h_{4}, 1\right)^{\alpha_{h}+\ell}}}
$$

for positive reals $\alpha_{g}, \alpha_{h}, \ell$ and

$$
\frac{\Gamma_{n, k}\left(g_{1}, \alpha \theta_{1}\right)^{\tau / \theta_{1}}-\Gamma_{n, k}\left(g_{2}, \alpha \theta_{2}\right)^{\tau / \theta_{2}}}{\Gamma_{n, k}\left(g_{3}, \alpha \theta_{3}\right)^{\tau / \theta_{3}}-\Gamma_{n, k}\left(g_{4}, \alpha \theta_{4}\right)^{\tau / \theta_{4}}} .
$$

for positive reals $\alpha, \theta_{i}$ and $\tau$. Note that the last-mentioned class is a weighted generalization of that of Fraga Alves et al. (2003).

The organization of the paper is as follows. In Section 2 we describe the hypotheses required on the underlying distribution $F$ and on the class of weighted functions $g$. Section 3 presents our results on the statistics $\Gamma_{n, k}(g, \alpha)$. We state weak consistency and asymptotic normality for the parameter $\gamma^{\alpha}$ in Proposition 1 and 2. In Corollary 1 and 2 we derive asymptotic properties of two estimators of the extreme value index. As a consequence, we propose an estimating procedure for large quantiles in Corollary 3. Section 4 is devoted to the estimation of (a function of) the second order parameter. Three different families of estimators are proved to be weak consistent in Proposition 4, 6 and 8. We also obtain the asymptotic normality of suitable normalization of these estimators in Proposition 5, 7 and 9. Section 5 gives some numerical results. Finally, all proofs are postponed to Section 6 .

\section{Preliminaries}

Let $X_{1}, \ldots, X_{n}$ be $n$ independent and identically distributed (i.i.d.) random variables with common continuous distribution function denoted by $F$. Let us denote by $X_{1, n} \leq \ldots \leq X_{n, n}$ the order statistics of our sample. Throughout the paper, our statistics of interest are $\Gamma_{n, k}(g, \alpha)$ given by $(2)$ with $\alpha$ some positive real and $g$ some well-behaved weighted function. In this section, we summarize assumptions required on $F$ and on the weighted function $g$. We also introduced some notations.

\subsection{Assumptions}

For $t>1$, we set

$$
U(t):=F^{\leftarrow}\left(1-\frac{1}{t}\right)
$$

where the arrow denotes the inverse function. Hypothesis (1) can be written in terms of the quantile function $U$ as follows.

Assumption $\left(\mathcal{H}_{1}\right)$ : There exists a positive real $\gamma$ such that

$$
\lim _{t \rightarrow \infty} \log U(t x)-\log U(t)=\gamma \log x, \quad \forall x>0 .
$$


In order to estimate the extreme value index $\gamma$ and more specifically to achieve the asymptotic normality of our estimators, we need to assume a second order expansion.

Assumption $\left(\mathcal{H}_{2}\right)$ : There exists a positive real $\gamma$, a negative real $\rho$ and a function $A$ with $A(t) \rightarrow 0$ of constant sign for large values of $t$ such that

$$
\lim _{t \rightarrow \infty} \frac{\log U(t x)-\log U(t)-\gamma \log x}{A(t)}=\frac{x^{\rho}-1}{\rho}, \quad \forall x>0 .
$$

Let us remark here that Assumption $\left(\mathcal{H}_{2}\right)$ implies that the function $|A|$ is regularly varying of index $\rho$ (see for instance de Haan and Ferreira, 2006).

As before, we can prove the asymptotic normality in the estimation of the second order parameter $\rho$ if a third order expansion is assumed.

Assumption $\left(\mathcal{H}_{3}\right)$ : There exists a positive real $\gamma$, some negative reals $\rho$ and $\beta$, some functions $A$ and $B$, with $A(t) \rightarrow 0$ and $B(t) \rightarrow 0$, both of constant sign for large values of $t$ such that

$$
\lim _{t \rightarrow \infty} \frac{(\log U(t x)-\log U(t)-\gamma \log x) / A(t)-\left(x^{\rho}-1\right) / \rho}{B(t)}=\frac{1}{\beta}\left(\frac{x^{\rho+\beta}-1}{\rho+\beta}-\frac{x^{\rho}-1}{\rho}\right), \quad \forall x>0 .
$$

Note that under Assumption $\left(\mathcal{H}_{3}\right)$ it can be proved that the function $|B|$ is regularly varying of index $\beta$. Once again we refer for details to de Haan and Ferreira (2006) and references therein.

We will assume that the weights satisfy the following condition.

Assumption $(\mathcal{G}): g$ is a positive, non-increasing and integrable function defined on $(0,1)$ such that there exists $\delta=\delta(g)>1 / 2$ satisfying $0<\int_{0}^{1} g(x) x^{-\delta} d x<\infty$ and $0<\int_{0}^{1} g(x)(1-x)^{-\delta} d x<\infty$.

Finally, throughout the paper we will assume that $k=k(n)$ is a sequence of integers satisfying Assumption $(\mathcal{K}): k \rightarrow \infty$ and $k / n \rightarrow 0$ as $n$ tends to infinity.

\subsection{Notations}

For the sake of clarity we introduce here some notations. Let $g$ and $\widetilde{g}$ be some functions defined on $(0,1)$. Let also $H$ and $\widetilde{H}$ be some well behaved functions in view of the definition of the following operators

$$
\begin{aligned}
\mu(g, H) & :=\int_{0}^{1} g(x) H(x) d x, \\
\nu(g, \widetilde{g}, H, \widetilde{H}) & :=\iint_{[0,1]^{2}} g(x) H^{\prime}(x) \widetilde{g}(y) \widetilde{H}^{\prime}(y)(x \wedge y)(1-x \vee y) d x d y, \\
\sigma^{2}(g, H) & :=\nu(g, g, H, H) .
\end{aligned}
$$

They will play the role of asymptotic mean, variance or covariance of some random variables.

For $x \in(0,1), \alpha$ some positive real, $\rho$ and $\beta$ some negative reals, let us denote

$$
\begin{aligned}
I_{\alpha}(x) & :=\left(\log x^{-1}\right)^{\alpha}, \\
J_{\alpha, \rho}(x) & :=\left(\log x^{-1}\right)^{\alpha-1} \frac{x^{-\rho}-1}{\rho}, \\
K_{\alpha, \rho}(x) & :=\left(\log x^{-1}\right)^{\alpha-2}\left(\frac{x^{-\rho}-1}{\rho}\right)^{2}, \\
L_{\alpha, \rho, \beta}(x) & :=\left(\log x^{-1}\right)^{\alpha-1} \frac{1}{\beta}\left(\frac{x^{-(\rho+\beta)}-1}{\rho+\beta}-\frac{x^{-\rho}-1}{\rho}\right) .
\end{aligned}
$$

Finally, when $H_{\alpha}$ and $\widetilde{H}_{\alpha}$ stand for some functions of the preceding list, we define the "normalized" version of the operators $\mu, \nu$ and $\sigma$ by

$$
\begin{aligned}
\bar{\mu}\left(g, H_{\alpha}\right) & :=\frac{\mu\left(g, H_{\alpha}\right)}{\mu\left(g, I_{\alpha}\right)} \\
\bar{\nu}\left(g, \widetilde{g}, H_{\alpha}, \widetilde{H}_{\alpha}\right) & :=\frac{\nu\left(g, \widetilde{g}, H_{\alpha}, \widetilde{H}_{\alpha}\right)}{\mu\left(g, I_{\alpha}\right) \mu\left(\widetilde{g}, I_{\alpha}\right)} \\
\bar{\sigma}^{2}\left(g, H_{\alpha}\right) & :=\bar{\nu}\left(g, g, H_{\alpha}, H_{\alpha}\right) .
\end{aligned}
$$




\section{Estimating the extreme value index}

In this part we present some generalizations for the estimation of the extreme value index $\gamma$. We start by the study of the asymptotic behavior of $\Gamma_{n, k}(g, \alpha)$ defined by $(2)$. We recall that $\alpha$ is assumed to be some positive real. As given in the next proposition, we see that under $\left(\mathcal{H}_{1}\right)$ these statistics form a family of weak consistent estimators of the parameter $\gamma^{\alpha}$.

Proposition 1. If $(\mathcal{G}),\left(\mathcal{H}_{1}\right)$ and $(\mathcal{K})$ hold then $\Gamma_{n, k}(g, \alpha) \rightarrow \gamma^{\alpha}$ in probability as $n \rightarrow \infty$.

When the second order expansion $\left(\mathcal{H}_{2}\right)$ holds true, we can derive the asymptotic normality property.

Proposition 2. If $(\mathcal{G}),\left(\mathcal{H}_{2}\right)$ and $(\mathcal{K})$ hold then we have the distributional representation

$$
\Gamma_{n, k}(g, \alpha)={ }^{d} \gamma^{\alpha}+\left(\gamma^{\alpha} \bar{\sigma}\left(g, I_{\alpha}\right) \frac{P_{k}(g, \alpha)}{\sqrt{k}}+A(n / k) \alpha \gamma^{\alpha-1} \bar{\mu}\left(g, J_{\alpha, \rho}\right)\right)\left(1+o_{\mathbb{P}}(1)\right)
$$

where $P_{k}(g, \alpha)$ is asymptotically standard normal.

As a consequence, if $\sqrt{k} A(n / k)$ tends to $\lambda<\infty$ as $n \rightarrow \infty$, we get

$$
\sqrt{k}\left(\Gamma_{n, k}(g, \alpha)-\gamma^{\alpha}\right) \stackrel{d}{\rightarrow} N\left(\lambda \alpha \gamma^{\alpha-1} \bar{\mu}\left(g, J_{\alpha, \rho}\right), \gamma^{2 \alpha} \bar{\sigma}^{2}\left(g, I_{\alpha}\right)\right) .
$$

Remark 1. As already remarked in the introduction, the statistics $\Gamma_{n, k}(g, \alpha)$ are shown to be generalizations over some estimators presented in the literature.

- $H_{n, k}:=\Gamma_{n, k}(1,1)$ corresponds to the Hill estimator (1975).

- $Z_{n, k}:=\Gamma_{n, k}\left(\log \left(\cdot{ }^{-1}\right)-1,1\right)$ is a slight modification of the QQ-estimator introduced by Kratz and Resnick (1996); Schultze and Steinebach (1996).

- For $\alpha>0, \Gamma_{n, k}(1, \alpha)$ are the statistics studied by Gomes and Martins $(1999,2001)$ and by Segers (2001).

- $\Gamma_{n, k}(g, 1)$ is the weighted least squares estimator of Hüsler et al. (2006) for positive $g$, of bounded variation on $(0,1)$ such that there exists $\delta=\delta(g)>0$ with $0<\int_{0}^{1} x^{-\delta} g(x) d x<\infty$ and $0<\int_{0}^{1}(1-x)^{-\delta} g(x) d x<\infty$.

Following the ideas of Gomes and Martins $(1999,2001)$ and Segers $(2001)$ we derive from $\Gamma_{n, k}(g, \alpha)$ some estimators of the parameter $\gamma$.

From the one hand, a possible estimator of the parameter $\gamma$ is $\Gamma_{n, k}^{1 / \alpha}(g, \alpha)$. Taking into account the distributional representation of $\Gamma_{n, k}(g, \alpha)$ given by (3), we obtain easily

Corollary 1. If $(\mathcal{G}),\left(\mathcal{H}_{2}\right)$ and $(\mathcal{K})$ hold, if moreover $\sqrt{k} A(n / k)$ tends to $\lambda<\infty$ as $n \rightarrow \infty$ then

$$
\sqrt{k}\left(\Gamma_{n, k}^{1 / \alpha}(g, \alpha)-\gamma\right) \stackrel{d}{\rightarrow} N\left(\lambda \bar{\mu}\left(g, J_{\alpha, \rho}\right), \frac{\gamma^{2}}{\alpha^{2}} \bar{\sigma}^{2}\left(g, I_{\alpha}\right)\right)
$$

From the other hand, another possible estimator of $\gamma$ is $\frac{\Gamma_{n, k}\left(g_{1}, \alpha\right)}{\Gamma_{n, k}^{\alpha-1}\left(g_{2}, 1\right)}$. Note the following result

Lemma 1. If $g_{1}$ and $g_{2}$ satisfy $(\mathcal{G})$ then $\left(P_{k}\left(g_{1}, \alpha_{1}\right), P_{k}\left(g_{2}, \alpha_{2}\right)\right)$ is asymptotically a centered bivariate normal random vector with covariance given by $\frac{\nu\left(g_{1}, g_{2}, I_{\alpha_{1}}, I_{\alpha_{2}}\right)}{\sigma\left(g_{1}, I_{\alpha_{1}}\right) \sigma\left(g_{2}, I_{\alpha_{2}}\right)}$.

Using the distributional representation of $\Gamma_{n, k}(g, \alpha)$ and Lemma 1 we obtain

Corollary 2. Assume that $g_{1}$ and $g_{2}$ satisfy $(\mathcal{G})$. If $\left(\mathcal{H}_{2}\right)$ and $(\mathcal{K})$ hold true and $\sqrt{k} A(n / k)$ tends to $\lambda<\infty$ as $n \rightarrow \infty$, then

$$
\sqrt{k}\left(\frac{\Gamma_{n, k}\left(g_{1}, \alpha\right)}{\Gamma_{n, k}^{\alpha-1}\left(g_{2}, 1\right)}-\gamma\right)
$$

is asymptotically normal with mean $\lambda\left[\alpha \bar{\mu}\left(g_{1}, J_{\alpha, \rho}\right)-(\alpha-1) \bar{\mu}\left(g_{2}, J_{1, \rho}\right)\right]$ and variance

$$
\gamma^{2}\left[\bar{\sigma}^{2}\left(g_{1}, I_{\alpha}\right)+(\alpha-1)^{2} \bar{\sigma}^{2}\left(g_{2}, I_{1}\right)-2(\alpha-1) \bar{\nu}\left(g_{1}, g_{2}, I_{\alpha}, I_{1}\right)\right] .
$$


A consequence of the estimation of the extreme index is that of high quantiles defined by $x_{p}:=F^{\leftarrow}(1-p)$ where the order $p$ tends to 0 . Several estimators of $x_{p}$ have been proposed in the literature by extrapolation along the fitted line of the Pareto QQ-plot. Indeed, Weissman (1978) proposed the well-known estimator

$$
\hat{x}_{n, k, p}^{H}:=X_{n-k, n}\left(\frac{k+1}{(n+1) p}\right)^{H_{k, n}}
$$

where we recall that $H_{n, k}$ denotes the Hill estimator. More recently, Fils and Guillou (2004) established the asymptotic behavior of the quantile estimator:

$$
\hat{x}_{n, k, p}^{Z}:=p^{-Z_{k, n}} \exp \left(\frac{1}{k} \sum_{i=1}^{k} \log X_{n-i+1, n}-\frac{Z_{k, n}}{k} \sum_{i=1}^{k} \log \left(\frac{n+1}{i}\right)\right)
$$

where we recall that $Z_{n, k}$ is the QQ-estimator. We deduce here the quantile estimator

$$
\hat{x}_{n, k, p}^{\Gamma}(g, \alpha):=X_{n-k, n}\left(\frac{k+1}{(n+1) p}\right)^{\Gamma_{n, k}^{1 / \alpha}(g, \alpha)} .
$$

Corollary 3. Under the assumption of Corollary 1 and for $p=p(n)$ such that $p$ and np tend to 0 as $n \rightarrow \infty$, we have

$$
\frac{\sqrt{k}}{\log a_{n}}\left(\log \hat{x}_{n, k, p}^{\Gamma}(g, \alpha)-\log x_{p}\right) \stackrel{d}{\rightarrow} N\left(\lambda \bar{\mu}\left(g, J_{\alpha, \rho}\right), \frac{\gamma^{2}}{\alpha^{2}} \bar{\sigma}^{2}\left(g, I_{\alpha}\right)\right)
$$

where $a_{n}=\frac{k+1}{(n+1) p}$.

\section{Estimating the second order parameter}

We derive in this part some estimators of the second order parameter from the statistics $\Gamma_{n, k}(g, \alpha)$. We start by a refinement of the distributional expansion.

Proposition 3. If $(\mathcal{G}),\left(\mathcal{H}_{3}\right)$ and $(\mathcal{K})$ hold then

$$
\begin{aligned}
\Gamma_{n, k}(g, \alpha) & ={ }^{d} \gamma^{\alpha}+\gamma^{\alpha} \bar{\sigma}\left(g, I_{\alpha}\right) \frac{P_{k}(g, \alpha)}{\sqrt{k}}+\alpha \gamma^{\alpha-1} \bar{\mu}\left(g, J_{\alpha, \rho}\right) A(n / k)+\alpha \gamma^{\alpha-1} \bar{\sigma}\left(g, J_{\alpha, \rho}\right) \bar{P}_{k}(g, \alpha, \rho) \frac{A(n / k)}{\sqrt{k}} \\
& +\frac{\alpha(\alpha-1)}{2} \gamma^{\alpha-2} \bar{\mu}\left(g, K_{\alpha, \rho}\right) A^{2}(n / k)\left(1+o_{\mathbb{P}}(1)\right)+\alpha \gamma^{\alpha-1} \bar{\mu}\left(g, L_{\alpha, \rho, \beta}\right) A(n / k) B(n / k)(1+o \mathbb{P}(1))
\end{aligned}
$$

where $\bar{P}_{k}(g, \alpha, \rho)$ is asymptotically standard normal.

Note that the third term in the distributional representation (5) is a function of the parameters $\gamma$ and $\rho$. The main idea consists in the combination (by difference and quotient) of several statistics $\Gamma_{n, k}(\cdot, \cdot)$ in order to get rid of the first two terms and to conserve only a weak consistent estimator of some function of $\rho$.

Throughout this section we consider a slight modification of the assumption on the sequence $k$ as follows. Assumption $(\widetilde{\mathcal{K}}): k \rightarrow \infty, k / n \rightarrow 0$ and $\sqrt{k} A(n / k) \rightarrow \infty$ as $n \rightarrow \infty$.

\subsection{First approach}

Let us denote $\boldsymbol{g}:=\left(g_{1}, g_{2}, g_{3}, g_{4}\right)$ for $g_{1}, g_{2}, g_{3}$ and $g_{4}$ some functions defined on $(0,1)$. For $\alpha_{1}$ and $\alpha_{2}$ some positive reals, we define the statistics

$$
\Psi_{n, k}\left(\boldsymbol{g}, \alpha_{1}, \alpha_{2}\right):=\frac{\Gamma_{n, k}\left(g_{1}, \alpha_{1}\right)-\Gamma_{n, k}\left(g_{3}, 1\right)^{\alpha_{1}}}{\Gamma_{n, k}\left(g_{2}, \alpha_{2}\right)-\Gamma_{n, k}\left(g_{4}, 1\right)^{\alpha_{2}}}
$$

and the function

$$
\psi\left(\boldsymbol{g}, \alpha_{1}, \alpha_{2}, \rho\right):=\frac{\alpha_{1}}{\alpha_{2}} \frac{\bar{\mu}\left(g_{1}, J_{\alpha_{1}, \rho}\right)-\bar{\mu}\left(g_{3}, J_{1, \rho}\right)}{\bar{\mu}\left(g_{2}, J_{\alpha_{2}, \rho}\right)-\bar{\mu}\left(g_{4}, J_{1, \rho}\right)} .
$$


Following the idea developed by Gomes et al. (2002), we propose to study the class of statistics

$$
S_{n, k}(\boldsymbol{g}, \boldsymbol{h}, \alpha, \ell):=\frac{\Psi_{n, k}(\boldsymbol{g}, 2 \alpha, 2 \ell)}{\left[\Psi_{n, k}(\boldsymbol{h}, \alpha+\ell, 2 \ell)\right]^{2}}
$$

where $\alpha$ and $\ell$ are some positive reals. The next proposition states that $S_{n, k}(\boldsymbol{g}, \boldsymbol{h}, \alpha, \ell)$ is a weak consistent estimator of the function of $\rho$ given by

$$
s(\boldsymbol{g}, \boldsymbol{h}, \alpha, \ell, \rho):=\frac{\psi(\boldsymbol{g}, 2 \alpha, 2 \ell, \rho)}{\psi^{2}(\boldsymbol{h}, \alpha+\ell, 2 \ell, \rho)} .
$$

Proposition 4. Assume that for $i=1, \ldots, 4$ the functions $g_{i}$ and $h_{i}$ satisfy Assumption $(\mathcal{G})$. Assume that $\left(\mathcal{H}_{2}\right)$ and $(\widetilde{\mathcal{K}})$ hold true. Suppose that $\psi(\boldsymbol{g}, 2 \alpha, 2 \ell, \rho)$ and $\psi(\boldsymbol{h}, \alpha+\ell, 2 \ell, \rho)$ are well defined and nonzero. Then $S_{n, k}(\boldsymbol{g}, \boldsymbol{h}, \alpha, \ell) \rightarrow s(\boldsymbol{g}, \boldsymbol{h}, \alpha, \ell, \rho)$ in probability as $n \rightarrow \infty$.

Now if we assume that the third order condition is true, then we can obtain the asymptotic distribution of a suitable normalization of $S_{n, k}(\boldsymbol{g}, \boldsymbol{h}, \alpha, \ell)-s(\boldsymbol{g}, \boldsymbol{h}, \alpha, \ell, \rho)$. Before, we need to introduce the notations

$$
\begin{aligned}
& m_{A}^{S}\left(\boldsymbol{g}, \alpha_{1}, \alpha_{2}, \rho\right):=\frac{\left(\alpha_{1}-1\right)\left[\bar{\mu}\left(g_{1}, K_{\alpha_{1}, \rho}\right)-\bar{\mu}^{2}\left(g_{3}, J_{1, \rho}\right)\right]-\left(\alpha_{2}-1\right)\left[\bar{\mu}\left(g_{2}, K_{\alpha_{2}, \rho}\right)-\bar{\mu}^{2}\left(g_{4}, J_{1, \rho}\right)\right] \psi\left(\boldsymbol{g}, \alpha_{1}, \alpha_{2}, \rho\right)}{\left(\bar{\mu}\left(g_{2}, J_{\alpha_{2}, \rho}\right)-\bar{\mu}\left(g_{4}, J_{1, \rho}\right)\right) \psi\left(\boldsymbol{g}, \alpha_{1}, \alpha_{2}, \rho\right)}, \\
& m_{B}^{S}\left(\boldsymbol{g}, \alpha_{1}, \alpha_{2}, \rho, \beta\right):=\frac{\left[\bar{\mu}\left(g_{1}, L_{\alpha_{1}, \rho, \beta}\right)-\bar{\mu}\left(g_{3}, L_{1, \rho, \beta}\right)\right]-\left[\bar{\mu}\left(g_{2}, L_{\alpha_{2}, \rho, \beta}\right)-\bar{\mu}\left(g_{4}, L_{1, \rho, \beta}\right)\right] \psi\left(\boldsymbol{g}, \alpha_{1}, \alpha_{2}, \rho\right)}{\left(\bar{\mu}\left(g_{2}, J_{\alpha_{2}, \rho}\right)-\bar{\mu}\left(g_{4}, J_{1, \rho}\right)\right) \psi\left(\boldsymbol{g}, \alpha_{1}, \alpha_{2}, \rho\right)}, \\
& \kappa_{S}\left(g_{1}, g_{2}, h_{1}, h_{2}, \alpha_{1}, \alpha_{2}\right):=\bar{\nu}\left(g_{1}, h_{1}, I_{\alpha_{1}}, I_{\alpha_{2}}\right)-\alpha_{2} \bar{\nu}\left(g_{1}, h_{2}, I_{\alpha_{1}}, I_{1}\right)-\alpha_{1} \bar{\nu}\left(g_{2}, h_{1}, I_{1}, I_{\alpha_{2}}\right)+\alpha_{1} \alpha_{2} \bar{\nu}\left(g_{2}, h_{2}, I_{1}, I_{1}\right), \\
& c_{S}\left(\boldsymbol{g}, \boldsymbol{h}, \alpha_{1}, \alpha_{2}, \alpha_{3}, \alpha_{4}, \rho\right):=\frac{1}{\alpha_{1} \alpha_{2} \alpha_{3} \alpha_{4}\left(\bar{\mu}\left(g_{2}, J_{\alpha_{2}, \rho}\right)-\bar{\mu}\left(g_{4}, J_{1, \rho}\right)\right) \psi\left(\boldsymbol{g}, \alpha_{1}, \alpha_{2}, \rho\right)\left(\bar{\mu}\left(h_{2}, J_{\alpha_{3}, \rho}\right)-\bar{\mu}\left(h_{4}, J_{1, \rho}\right)\right) \psi\left(\boldsymbol{h}, \alpha_{3}, \alpha_{4}, \rho\right)} \\
& \quad \times\left[\alpha_{2} \alpha_{4} \kappa_{S}\left(g_{1}, g_{3}, h_{1}, h_{3}, \alpha_{1}, \alpha_{3}\right)-\alpha_{2} \alpha_{3} \psi\left(\boldsymbol{h}, \alpha_{3}, \alpha_{4}, \rho\right) \kappa_{S}\left(g_{1}, g_{3}, h_{2}, h_{4}, \alpha_{1}, \alpha_{4}\right)-\right. \\
& \left.\quad \alpha_{1} \alpha_{4} \psi\left(\boldsymbol{g}, \alpha_{1}, \alpha_{2}, \rho\right) \kappa_{S}\left(g_{2}, g_{4}, h_{1}, h_{3}, \alpha_{2}, \alpha_{3}\right)+\alpha_{1} \alpha_{3} \psi\left(\boldsymbol{g}, \alpha_{1}, \alpha_{2}, \rho\right) \psi\left(\boldsymbol{h}, \alpha_{3}, \alpha_{4}, \rho\right) \kappa_{S}\left(g_{2}, g_{4}, h_{2}, h_{4}, \alpha_{2}, \alpha_{4}\right)\right] \\
& v_{S}^{2}\left(\boldsymbol{g}, \alpha_{1}, \alpha_{2}, \rho\right):=c_{S}\left(\boldsymbol{g}, \boldsymbol{g}, \alpha_{1}, \alpha_{2}, \alpha_{1}, \alpha_{2}, \rho\right)
\end{aligned}
$$

Proposition 5. Assume that for $i=1, \ldots, 4$ the functions $g_{i}$ and $h_{i}$ satisfy Assumption $(\mathcal{G})$. Assume that $\left(\mathcal{H}_{3}\right)$ and $(\widetilde{\mathcal{K}})$ hold true with $\sqrt{k} A^{2}(n / k) \rightarrow \lambda_{A}<\infty$ and $\sqrt{k} A(n / k) B(n / k) \rightarrow \lambda_{B}<\infty$ as $n \rightarrow \infty$. Suppose that $\psi(\boldsymbol{g}, 2 \alpha, 2 \ell, \rho)$ and $\psi(\boldsymbol{h}, \alpha+\ell, 2 \ell, \rho)$ are well defined and nonzero. Then

$$
\sqrt{k} A(n / k)\left(S_{n, k}(\boldsymbol{g}, \boldsymbol{h}, \alpha, \ell)-s(\boldsymbol{g}, \boldsymbol{h}, \alpha, \ell, \rho)\right)
$$

is asymptotically a normal random variable with mean

$$
s(\boldsymbol{g}, \boldsymbol{h}, \alpha, \ell, \rho)\left(\lambda_{A} \frac{m_{A}^{S}(\boldsymbol{g}, 2 \alpha, 2 \ell, \rho)-2 m_{A}^{S}(\boldsymbol{h}, \alpha+\ell, 2 \ell, \rho)}{2 \gamma}+\lambda_{B}\left(m_{B}^{S}(\boldsymbol{g}, 2 \alpha, 2 \ell, \rho, \beta)-2 m_{B}^{S}(\boldsymbol{h}, \alpha+\ell, 2 \ell, \rho, \beta)\right)\right)
$$

and variance

$$
\gamma^{2} s^{2}(\boldsymbol{g}, \boldsymbol{h}, \alpha, \ell, \rho)\left(v_{S}^{2}(\boldsymbol{g}, 2 \alpha, 2 \ell, \rho)+4 v_{S}^{2}(\boldsymbol{h}, \alpha+\ell, 2 \ell, \rho)-4 c_{S}(\boldsymbol{g}, \boldsymbol{h}, 2 \alpha, 2 \ell, \alpha+\ell, 2 \ell, \rho)\right) .
$$

\subsection{Second approach}

For $\alpha_{g}, \alpha_{h}$ and $\ell$ some positive reals, we introduce the family of statistics

$$
Q_{n, k}\left(\boldsymbol{g}, \boldsymbol{h}, \alpha_{g}, \alpha_{h}, \ell\right):=\frac{\Psi_{n, k}\left(\boldsymbol{g}, \alpha_{g}, \alpha_{g}+\ell\right)}{\Psi_{n, k}\left(\boldsymbol{h}, \alpha_{h}, \alpha_{h}+\ell\right)}
$$

and the function

$$
q\left(\boldsymbol{g}, \boldsymbol{h}, \alpha_{g}, \alpha_{h}, \ell, \rho\right):=\frac{\psi\left(\boldsymbol{g}, \alpha_{g}, \alpha_{g}+\ell, \rho\right)}{\psi\left(\boldsymbol{h}, \alpha_{h}, \alpha_{h}+\ell, \rho\right)} .
$$

This construction is clearly inspired by the preceding one. However it does not appear in the literature since for constant weighted functions $\boldsymbol{g} \equiv \boldsymbol{h} \equiv(1,1,1,1)$, the limit $q\left(\boldsymbol{g}, \boldsymbol{h}, \alpha_{g}, \alpha_{h}, \ell, \rho\right)$ does not depend on $\rho$. As previously, the second order condition allows us to prove that $Q_{n, k}\left(\boldsymbol{g}, \boldsymbol{h}, \alpha_{g}, \alpha_{h}, \ell\right)$ is a weak consistent estimator of $q\left(\boldsymbol{g}, \boldsymbol{h}, \alpha_{g}, \alpha_{h}, \ell, \rho\right)$. 
Proposition 6. Assume that for $i=1, \ldots, 4$ the functions $g_{i}$ and $h_{i}$ satisfy Assumption $(\mathcal{G})$. Assume that $\left(\mathcal{H}_{2}\right)$ and $(\widetilde{\mathcal{K}})$ hold true. Suppose that $\psi\left(\boldsymbol{g}, \alpha_{g}, \alpha_{g}+\ell, \rho\right)$ and $\psi\left(\boldsymbol{h}, \alpha_{h}, \alpha_{h}+\ell, \rho\right)$ are well defined and nonzero. Then $Q_{n, k}\left(\boldsymbol{g}, \boldsymbol{h}, \alpha_{g}, \alpha_{h}, \ell\right) \rightarrow q\left(\boldsymbol{g}, \boldsymbol{h}, \alpha_{g}, \alpha_{h}, \ell, \rho\right)$ in probability as $n \rightarrow \infty$.

Moreover, taking into account the notations defined in Section 4.2, we obtain

Proposition 7. Assume that for $i=1, \ldots, 4$ the functions $g_{i}$ and $h_{i}$ satisfy Assumption $(\mathcal{G})$. Assume that $\left(\mathcal{H}_{3}\right)$ and $(\widetilde{\mathcal{K}})$ hold true with $\sqrt{k} A^{2}(n / k) \rightarrow \lambda_{A}<\infty$ and $\sqrt{k} A(n / k) B(n / k) \rightarrow \lambda_{B}<\infty$ as $n \rightarrow \infty$. Suppose that $\psi\left(\boldsymbol{g}, \alpha_{g}, \alpha_{g}+\ell, \rho\right)$ and $\psi\left(\boldsymbol{h}, \alpha_{h}, \alpha_{h}+\ell, \rho\right)$ are well defined and nonzero. Then

$$
\sqrt{k} A(n / k)\left(Q_{n, k}\left(\boldsymbol{g}, \boldsymbol{h}, \alpha_{g}, \alpha_{h}, \ell\right)-q\left(\boldsymbol{g}, \boldsymbol{h}, \alpha_{g}, \alpha_{h}, \ell, \rho\right)\right)
$$

is asymptotically a normal random variable with mean

$$
q\left(\boldsymbol{g}, \boldsymbol{h}, \alpha_{g}, \alpha_{h}, \ell, \rho\right)\left(\lambda_{A} \frac{m_{A}^{S}\left(\boldsymbol{g}, \alpha_{g}, \alpha_{g}+\ell, \rho\right)-m_{A}^{S}\left(\boldsymbol{h}, \alpha_{h}, \alpha_{h}+\ell, \rho\right)}{2 \gamma}+\lambda_{B}\left(m_{B}^{S}\left(\boldsymbol{g}, \alpha_{g}, \alpha_{g}+\ell, \rho, \beta\right)-m_{B}^{S}\left(\boldsymbol{h}, \alpha_{h}, \alpha_{h}+\ell, \rho, \beta\right)\right)\right)
$$

and variance

$$
\gamma^{2} q^{2}\left(\boldsymbol{g}, \boldsymbol{h}, \alpha_{g}, \alpha_{h}, \ell, \rho\right)\left(v_{S}^{2}\left(\boldsymbol{g}, \alpha_{g}, \alpha_{g}+\ell, \rho\right)+v_{S}^{2}\left(\boldsymbol{h}, \alpha_{h}, \alpha_{h}+\ell, \rho\right)-2 c_{S}\left(\boldsymbol{g}, \boldsymbol{h}, \alpha_{g}, \alpha_{g}+\ell, \alpha_{h}, \alpha_{h}+\ell, \rho\right)\right) .
$$

\subsection{Third approach}

For $\theta_{1}, \theta_{2}, \theta_{3}$ and $\theta_{4}$ some positive reals, let us denote $\boldsymbol{\theta}:=\left(\theta_{1}, \theta_{2}, \theta_{3}, \theta_{4}\right)$. Let also $\tau$ denotes some positive real. Following the construction of Fraga Alves et al. (2003) we consider the statistics

$$
\Phi_{n, k}(\boldsymbol{g}, \alpha, \boldsymbol{\theta}, \tau):=\frac{\Gamma_{n, k}\left(g_{1}, \alpha \theta_{1}\right)^{\tau / \theta_{1}}-\Gamma_{n, k}\left(g_{2}, \alpha \theta_{2}\right)^{\tau / \theta_{2}}}{\Gamma_{n, k}\left(g_{3}, \alpha \theta_{3}\right)^{\tau / \theta_{3}}-\Gamma_{n, k}\left(g_{4}, \alpha \theta_{4}\right)^{\tau / \theta_{4}}} .
$$

and the function

$$
\phi(\boldsymbol{g}, \alpha, \boldsymbol{\theta}, \rho):=\frac{\bar{\mu}\left(g_{1}, J_{\alpha \theta_{1}, \rho}\right)-\bar{\mu}\left(g_{2}, J_{\alpha \theta_{2}, \rho}\right)}{\bar{\mu}\left(g_{3}, J_{\alpha \theta_{3}, \rho}\right)-\bar{\mu}\left(g_{4}, J_{\alpha \theta_{4}, \rho}\right)} .
$$

This class of estimators has clearly the simplest form.

Proposition 8. Assume that $g_{1}, g_{2}, g_{3}$ and $g_{4}$ satisfy Assumption $(\mathcal{G})$. Assume that $\left(\mathcal{H}_{2}\right)$ and $(\widetilde{\mathcal{K}})$ hold true. Suppose that $\phi(\boldsymbol{g}, \alpha, \boldsymbol{\theta}, \rho)$ is well defined and nonzero. Then $\Phi_{n, k}(\boldsymbol{g}, \alpha, \boldsymbol{\theta}, \tau) \rightarrow \phi(\boldsymbol{g}, \alpha, \boldsymbol{\theta}, \rho)$ in probability as $n \rightarrow \infty$.

In order to describe the asymptotic normality associated to this estimator, we need to introduce some notations.

$$
\begin{aligned}
& m_{\Phi}^{A}\left(g_{1}, g_{2}, \alpha_{1}, \alpha_{2}, \alpha_{3}, \rho\right):=\left(\alpha_{1}-1\right) \bar{\mu}\left(g_{1}, K_{\alpha_{1}, \rho}\right)+\left(\alpha_{3}-\alpha_{1}\right) \bar{\mu}^{2}\left(g_{1}, J_{\alpha_{1}, \rho}\right)-\left(\alpha_{2}-1\right) \bar{\mu}\left(g_{2}, K_{\alpha_{2}, \rho}\right)-\left(\alpha_{3}-\alpha_{2}\right) \bar{\mu}^{2}\left(g_{2}, J_{\alpha_{2}, \rho}\right), \\
& m_{\Phi}^{B}\left(g_{1}, g_{2}, \alpha_{1}, \alpha_{2}, \rho, \beta\right):=\bar{\mu}\left(g_{1}, L_{\alpha_{1}, \rho, \beta}\right)-\bar{\mu}\left(g_{2}, L_{\alpha_{2}, \rho, \beta}\right), \\
& c_{\Phi}\left(g_{1}, g_{2}, g_{3}, g_{4}, \alpha_{1}, \alpha_{2}, \alpha_{3}, \alpha_{4}\right):=\frac{\bar{\nu}\left(g_{1}, g_{3}, I_{\alpha_{1}}, I_{\alpha_{3}}\right)}{\alpha_{1} \alpha_{3}}-\frac{\bar{\nu}\left(g_{1}, g_{4}, I_{\alpha_{1}}, I_{\alpha_{4}}\right)}{\alpha_{1} \alpha_{4}}-\frac{\bar{\nu}\left(g_{2}, g_{3}, I_{\alpha_{2}}, I_{\alpha_{3}}\right)}{\alpha_{2} \alpha_{3}}+\frac{\bar{\nu}\left(g_{2}, g_{4}, I_{\alpha_{2}}, I_{\alpha_{4}}\right)}{\alpha_{2} \alpha_{4}}, \\
& v_{\Phi}^{2}\left(g_{1}, g_{2}, \alpha_{1}, \alpha_{2}\right):=c_{\Phi}\left(g_{1}, g_{2}, g_{1}, g_{2}, \alpha_{1}, \alpha_{2}, \alpha_{1}, \alpha_{2}\right) .
\end{aligned}
$$

Proposition 9. Assume that $g_{1}, g_{2}, g_{3}$ and $g_{4}$ satisfy Assumption $(\mathcal{G})$ with $\bar{\mu}\left(g_{3}, L_{\alpha \theta_{3}, \rho}\right) \neq \bar{\mu}\left(g_{4}, L_{\alpha \theta_{4}, \rho}\right)$. Assume that $\left(\mathcal{H}_{3}\right)$ and $(\widetilde{\mathcal{K}})$ hold true with $\sqrt{k} A^{2}(n / k) \rightarrow \lambda_{A}<\infty$ and $\sqrt{k} A(n / k) B(n / k) \rightarrow \lambda_{B}<\infty$ as $n \rightarrow \infty$. Then

$$
\sqrt{k} A(n / k)\left(\Phi_{n, k}(\boldsymbol{g}, \alpha, \boldsymbol{\theta}, \tau)-\phi(\boldsymbol{g}, \alpha, \boldsymbol{\theta}, \rho)\right)
$$

is asymptotically a normal random variable with mean

$\lambda_{A} \frac{m_{\Phi}^{A}\left(g_{1}, g_{2}, \alpha \theta_{1}, \alpha \theta_{2}, \alpha \tau, \rho\right)-m_{\Phi}^{A}\left(g_{3}, g_{4}, \alpha \theta_{3}, \alpha \theta_{4}, \alpha \tau, \rho\right) \phi(\boldsymbol{g}, \alpha, \boldsymbol{\theta}, \rho)}{2 \gamma\left(\bar{\mu}\left(g_{3}, J_{\alpha \theta_{3}, \rho}\right)-\bar{\mu}\left(g_{4}, J_{\alpha \theta_{4}, \rho}\right)\right)}+\lambda_{B} \frac{m_{\Phi}^{B}\left(g_{1}, g_{2}, \alpha \theta_{1}, \alpha \theta_{2}, \rho, \beta\right)-m_{\Phi}^{B}\left(g_{3}, g_{4}, \alpha \theta_{1}, \alpha \theta_{2}, \rho, \beta\right) \phi(\boldsymbol{g}, \alpha, \boldsymbol{\theta}, \rho)}{\bar{\mu}\left(g_{3}, J_{\alpha \theta_{3}, \rho}\right)-\bar{\mu}\left(g_{4}, J_{\alpha \theta_{4}, \rho}\right)}$

and variance

$$
\gamma^{2} \frac{v_{\Phi}^{2}\left(g_{1}, g_{2}, \alpha \theta_{1}, \alpha \theta_{2}\right)+v_{\Phi}^{2}\left(g_{3}, g_{4}, \alpha \theta_{3}, \alpha \theta_{4}\right) \phi^{2}(\boldsymbol{g}, \alpha, \boldsymbol{\theta}, \rho)-2 c_{\Phi}\left(g_{1}, g_{2}, g_{3}, g_{4}, \alpha \theta_{1}, \alpha \theta_{2}, \alpha \theta_{3}, \alpha \theta_{4}\right) \phi(\boldsymbol{g}, \alpha, \boldsymbol{\theta}, \rho)}{\left(\bar{\mu}\left(g_{3}, J_{\alpha \theta_{3}, \rho}\right)-\bar{\mu}\left(g_{4}, J_{\alpha \theta_{4}, \rho}\right)\right)^{2}} .
$$

Remark 2. If $\tau$ tends to 0 then the statistic $\Phi_{n, k}(\boldsymbol{g}, \alpha, \boldsymbol{\theta}, \tau)$ tends to

$$
\Phi_{n, k}(\boldsymbol{g}, \alpha, \boldsymbol{\theta}, 0):=\frac{\left(\log \Gamma_{n, k}\left(g_{1}, \alpha \theta_{1}\right)\right) / \theta_{1}-\left(\log \Gamma_{n, k}\left(g_{2}, \alpha \theta_{2}\right)\right) / \theta_{2}}{\left(\log \Gamma_{n, k}\left(g_{3}, \alpha \theta_{3}\right)\right) / \theta_{3}-\left(\log \Gamma_{n, k}\left(g_{4}, \alpha \theta_{4}\right)\right) / \theta_{4}} .
$$

Moreover, it can be proved that Propositions 8 and 9 hold true setting $\tau=0$ everywhere. 


\subsection{Consequences for the estimation of $\rho$}

These approaches furnish three consistent classes of estimators of some function of $\rho$. As a consequence, it is also possible to derive associated class of estimators of $\rho$ by inversion. Assume that we can choose weighted functions and tuning parameters such that the functions $\rho \mapsto s(\boldsymbol{g}, \boldsymbol{h}, \alpha, \ell, \rho), \rho \mapsto q\left(\boldsymbol{g}, \boldsymbol{h}, \alpha_{g}, \alpha_{h}, \ell, \rho\right)$ and $\rho \mapsto \phi(\boldsymbol{g}, \alpha, \boldsymbol{\theta}, \rho)$ are bijective. Let us denote by $s^{\leftarrow}(\boldsymbol{g}, \boldsymbol{h}, \alpha, \ell, \cdot), q^{\leftarrow}\left(\boldsymbol{g}, \boldsymbol{h}, \alpha_{g}, \alpha_{h}, \ell, \cdot\right)$ and $\phi^{\leftarrow}(\boldsymbol{g}, \alpha, \boldsymbol{\theta}, \cdot)$ their inverse functions. Then we can define

$$
\begin{aligned}
\hat{\rho}_{n, k}^{S}(\boldsymbol{g}, \boldsymbol{h}, \alpha, \ell) & :=s^{\leftarrow}\left(\boldsymbol{g}, \boldsymbol{h}, \alpha, \ell, S_{n, k}(\boldsymbol{g}, \boldsymbol{h}, \alpha, \ell)\right), \\
\hat{\rho}_{n, k}^{Q}\left(\boldsymbol{g}, \boldsymbol{h}, \alpha_{g}, \alpha_{h}, \ell\right) & :=q^{\leftarrow}\left(\boldsymbol{g}, \boldsymbol{h}, \alpha_{g}, \alpha_{h}, \ell, Q_{n, k}\left(\boldsymbol{g}, \boldsymbol{h}, \alpha_{g}, \alpha_{h}, \ell\right)\right), \\
\hat{\rho}_{n, k}^{\Phi}(\boldsymbol{g}, \alpha, \boldsymbol{\theta}, \tau) & :=\phi^{\leftarrow}\left(\boldsymbol{g}, \alpha, \boldsymbol{\theta}, \Phi_{n, k}(\boldsymbol{g}, \alpha, \boldsymbol{\theta}, \tau)\right) .
\end{aligned}
$$

Moreover, assume that these inverse functions are continuous. By application of the Continuous mapping Theorem, under the assumptions of Proposition 4 (resp. 6 and 8) it can be proved that $\hat{\rho}_{n, k}^{S}(\boldsymbol{g}, \boldsymbol{h}, \alpha, \ell)$ (resp. $\hat{\rho}_{n, k}^{Q}\left(\boldsymbol{g}, \boldsymbol{h}, \alpha_{g}, \alpha_{h}, \ell\right)$ and $\left.\hat{\rho}_{n, k}^{\Phi}(\boldsymbol{g}, \alpha, \boldsymbol{\theta}, \tau)\right)$ is weak consistent for the parameter $\rho$. Finally, if moreover these inverse functions are differentiable, the asymptotic normality of the statistics suitably normalized may be obtained from the Delta method under the assumptions of Proposition 5, 7 and 9 respectively.

Some examples, derived from the third approach, are presented in Section 5.2.

\section{$5 \quad$ Numerical results}

In this part we show numerical results for the estimation of the parameters $\gamma$ and $\rho$ in finite-sample and asymptotic framework respectively.

\subsection{Simulations for the estimation of the extreme value index}

To illustrate the finite-sample behavior of some estimators studied in this paper we give some simulation results for the distribution functions given in Table 1 and the weighted functions given in Table 2.

\begin{tabular}{lll}
\hline Distributions & $\gamma$ & $\rho$ \\
\hline Standard Cauchy & 1 & -2 \\
Fréchet(2) & 2 & -1 \\
\hline
\end{tabular}

Table 1: Tail characteristics of some distribution functions.

\begin{tabular}{cc}
\hline Weighted functions $g$ & Expressions \\
\hline$g_{0}$ & 1 \\
$g_{1}$ & $2(1-x)$ \\
$g_{2}$ & $3 / 2\left(1-x^{2}\right)$ \\
$g_{3}$ & $\log x^{-1}$ \\
\hline
\end{tabular}

Table 2: Weighted functions satisfying Assumption $(\mathcal{G})$.

Figure 1, 2 and 3 are obtained as follows. We generate independent and identically distributed samples of size $n=1000$ and replicate them $m=5000$ times independently. For the sake of simplicity, let us denote the estimates by $\hat{\gamma}_{1}, \ldots, \hat{\gamma}_{m}$. We compute the mean $1 / m \sum_{i=1}^{m} \hat{\gamma}_{i}$ and the mean square error $1 / m \sum_{i=1}^{m}\left(\hat{\gamma}_{i}-\gamma\right)^{2}$. Since all the estimators depend on $k$ upper order statistics, we show these averages as functions of $k$.

We present the results for the Standard Cauchy distribution on the left and the Fréchet(2) distribution on the right. We use on the same sample the estimators

(1) $\Gamma_{n, k}\left(g_{i}, 1\right)$ for $i \in\{0,1,2,3\}$ in Figure 1 ,

(2) $\Gamma_{n, k}\left(g_{i}, \alpha\right)^{1 / \alpha}$ for $i \in\{0,1\}$ and $\alpha \in\{0.5,2\}$ in Figure 2,

(3) $\Gamma_{n, k}\left(g_{i}, \alpha\right) / \Gamma_{n, k}^{\alpha-1}\left(g_{0}, 1\right)$ for $i \in\{1,2\}$ and $\alpha \in\{0.5,2\}$ in Figure 3. 

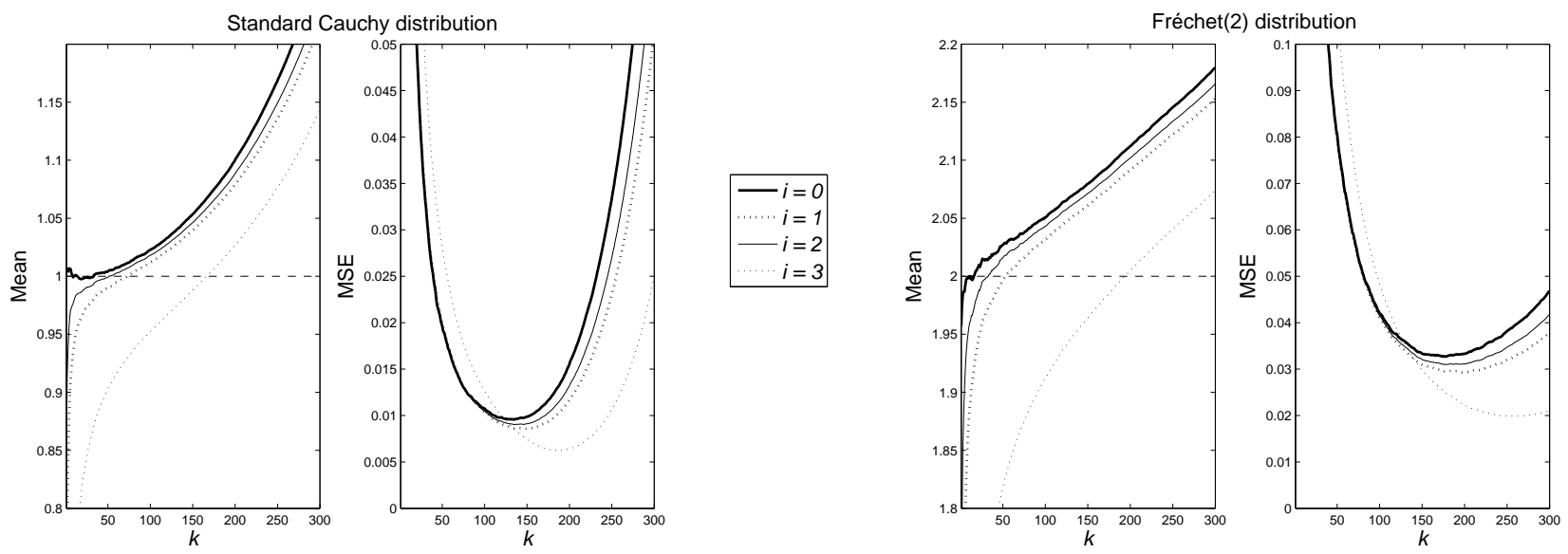

Figure 1: Mean and MSE in the case (1).
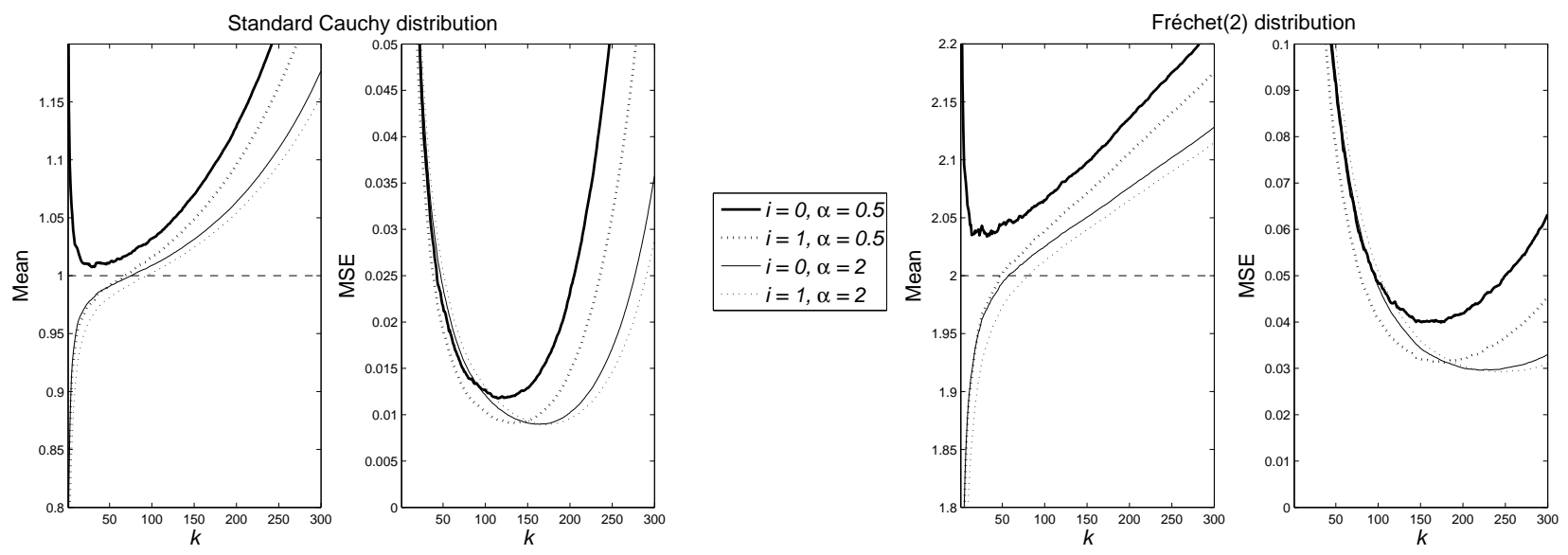

Figure 2: Mean and MSE in the case (2).
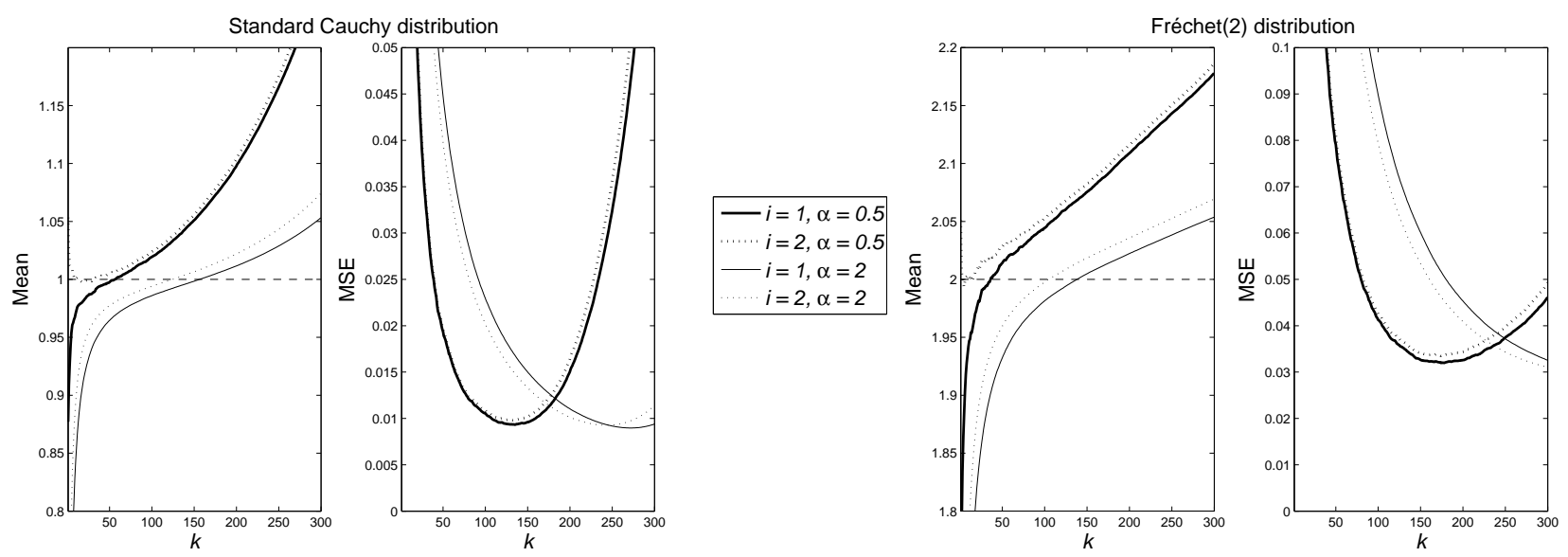

Figure 3: Mean and MSE in the case (3). 


\subsection{Asymptotic properties in the estimation of the second order parameter}

We present here four estimators of $\rho$ constructed from the third approach explained in Section 4.3. Let us consider the family $\Phi_{n, k}(\boldsymbol{g}, \alpha, \boldsymbol{\theta}, \tau)$ for $\alpha=1$ and particular values of $\boldsymbol{g}$ and $\boldsymbol{\theta}$

$$
\begin{aligned}
\Phi_{n, k}^{[1]}(\tau) & :=\Phi_{n, k}\left(\left(g_{0}, g_{0}, g_{0}, g_{0}\right), 1,(1,2,2,3), \tau\right), \\
\Phi_{n, k}^{[2]}(\tau) & :=\Phi_{n, k}\left(\left(g_{1}, g_{0}, g_{0}, g_{2}\right), 1,(1,1,1,1), \tau\right), \\
\Phi_{n, k}^{[3]}(\tau) & :=\Phi_{n, k}\left(\left(g_{1}, g_{0}, g_{0}, g_{1}\right), 1,(1,2,1,1), \tau\right), \\
\Phi_{n, k}^{[4]}(\tau) & :=\Phi_{n, k}\left(\left(g_{1}, g_{0}, g_{2}, g_{1}\right), 1,(1,1,1,1), \tau\right) .
\end{aligned}
$$

Recall that the weighted functions $g_{i}$ are given in Table 2. Under the assumptions of Proposition 8, these statistics converge in probability (respectively and independently of $\tau$ ) towards

$$
\begin{aligned}
\phi\left(\left(g_{0}, g_{0}, g_{0}, g_{0}\right), 1,(1,2,2,3), \rho\right) & =\frac{3(1-\rho)}{3-\rho} \\
\phi\left(\left(g_{1}, g_{0}, g_{0}, g_{2}\right), 1,(1,1,1,1), \rho\right) & =-\frac{4}{3} \frac{3-\rho}{2-\rho} \\
\phi\left(\left(g_{1}, g_{0}, g_{0}, g_{1}\right), 1,(1,2,1,1), \rho\right) & =\frac{1}{2} \frac{4-\rho}{1-\rho} \\
\phi\left(\left(g_{1}, g_{0}, g_{2}, g_{1}\right), 1,(1,1,1,1), \rho\right) & =-\frac{4(3-\rho)}{6-\rho} .
\end{aligned}
$$

By inversion, we obtain the following weak consistent estimators of $\rho$

$$
\begin{aligned}
& \hat{\rho}_{n, k}^{[1]}(\tau):=\frac{3\left(\Phi_{n, k}^{[1]}(\tau)-1\right)}{\Phi_{n, k}^{[1]}(\tau)-3} \quad \text { provided that } 1 \leq \Phi_{n, k}^{[1]}(\tau)<3, \\
& \hat{\rho}_{n, k}^{[2]}(\tau):=\frac{6\left(\Phi_{n, k}^{[2]}(\tau)+2\right)}{3 \Phi_{n, k}^{[2]}(\tau)+4} \quad \text { provided that }-2 \leq \Phi_{n, k}^{[2]}(\tau)<-4 / 3, \\
& \hat{\rho}_{n, k}^{[3]}(\tau):=\frac{2\left(\Phi_{n, k}^{[3]}(\tau)-2\right)}{2 \Phi_{n, k}^{[3]}(\tau)-1} \quad \text { provided that } 1 / 2<\Phi_{n, k}^{[3]}(\tau) \leq 2, \\
& \hat{\rho}_{n, k}^{[4]}(\tau):=\frac{6\left(\Phi_{n, k}^{[4]}(\tau)+2\right)}{\Phi_{n, k}^{[4]}(\tau)+4} \quad \text { provided that }-4<\Phi_{n, k}^{[4]}(\tau) \leq-2,
\end{aligned}
$$

where the conditions yield the right sign for the estimators. Then, under the assumptions of Proposition 8 , we have for $i=1, \ldots, 4$

$$
\sqrt{k} A(n / k)\left(\hat{\rho}_{n, k}^{[i]}(\tau)-\rho\right) \underset{n \rightarrow \infty}{\stackrel{d}{\longrightarrow}} N\left(\frac{\lambda_{A}}{\gamma} m_{A,[i]}(\tau, \rho)+\lambda_{B} m_{B,[i]}(\rho, \beta), \gamma^{2} v_{[i]}^{2}(\rho)\right) .
$$

Straightforward computations give the expressions of the asymptotic variance components

$$
\begin{aligned}
& v_{[1]}^{2}(\rho)=\frac{(1-\rho)^{6}}{\rho^{2}}\left(1-2 \rho+2 \rho^{2}\right), \\
& v_{[2]}^{2}(\rho)=\frac{(2-\rho)^{2}\left(3-4 \rho+\rho^{2}\right)^{2}}{27 \rho^{2}}\left(36-12 \rho+\rho^{2}\right), \\
& v_{[3]}^{2}(\rho)=\frac{(1-\rho)^{4}(2-\rho)^{2}}{3 \rho^{2}}\left(1-\rho+\rho^{2}\right), \\
& v_{[4]}^{2}(\rho)=\frac{(3-\rho)^{2}\left(2-3 \rho+\rho^{2}\right)^{2}}{30 \rho^{2}}\left(4-\rho+\rho^{2}\right) .
\end{aligned}
$$

In Figure 4, we plot for $i=1, \ldots, 4$ the asymptotic component $v_{[i]}(\rho), m_{A,[i]}(\tau, \rho)$ for $\tau \in\{0,1\}$ and $m_{B,[i]}(\rho, \beta)$ for $\beta \in\{-2,-1\}$ as functions of $\rho \in(-5 / 2,0)$. The cases $i=2$ and $i=4$ give the same results for the asymptotic mean components. 


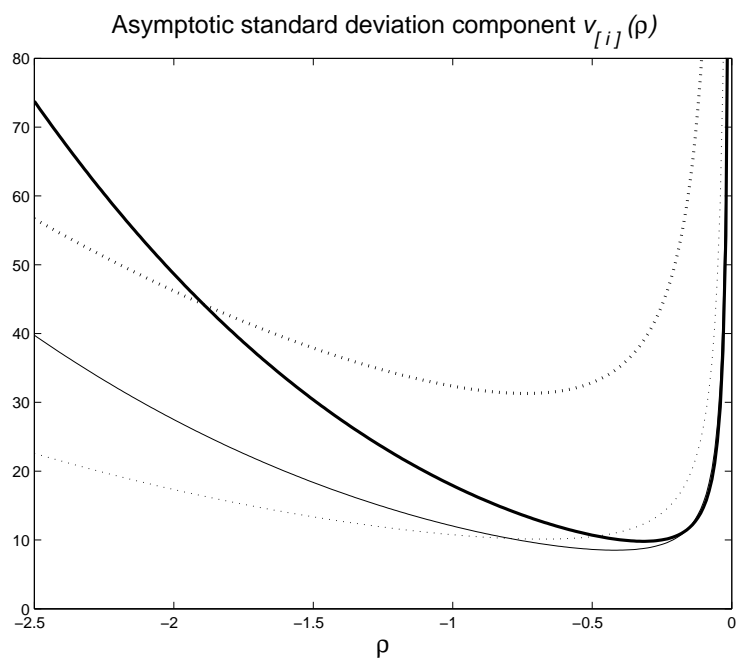

$-i=1 \cdots \cdots+i=3 \cdots \cdots i=4$


Figure 4: Behavior of the asymptotic mean and standard deviation components. 


\section{Proofs}

\subsection{Main tool}

We present here the essential tool in proving our results. For convenience we set

$$
M_{n, k}(g, \alpha):=\frac{1}{k} \sum_{i=1}^{k} g\left(\frac{i}{k+1}\right)\left[\log \frac{X_{n-i+1, n}}{X_{n-k, n}}\right]^{\alpha} .
$$

Let $U_{i, k}$ denote the ith-order statistic of a standard uniform sample of size $k$ and by $H$ some function defined on $(0,1)$. Under classical assumptions, the statistics $M_{n, k}(g, \alpha)$ can be written (in the sense of distributional representation) as linear combinations of terms with the following form

$$
\frac{1}{k} \sum_{i=1}^{k} g(i /(k+1)) H\left(U_{i, k}\right)
$$

The asymptotic behavior of such random variables has been studied by Chernoff et al. (1967). Consequently, we recall here a slightly simplified version of their Theorem 3.

Theorem 1. Let $H$ be a continuous function on $(0,1)$, satisfying a first order Lipschitz condition in every interval bounded away from 0 and 1 , whose derivative $H^{\prime}$ exists and is continuous except on a set $S$ of Jordan content 0 . Assume that there exists some $\tau_{0} \in(0,1)$ such that

$\star \forall K>0, \exists M<\infty$ s.t. if $0<u_{1}, u_{2}<\tau_{0}$ and $K^{-1}<\frac{u_{1}}{u_{2}}<K$ then $M^{-1}<\frac{H^{\prime}\left(u_{1}\right)}{H^{\prime}\left(u_{2}\right)}<M$,

$\star \forall K>0, \exists M<\infty$ s.t. if $1-\tau_{0}<u_{1}, u_{2}<1$ and $K^{-1}<\frac{1-u_{1}}{1-u_{2}}<K$ then $M^{-1}<\frac{H^{\prime}\left(u_{1}\right)}{H^{\prime}\left(u_{2}\right)}<M$.

Let now $g$ be a function defined on $(0,1)$ such that

(i) $\int_{\theta}^{1-\theta} g(x) d x$ converges absolutely for any $\theta \in(0,1 / 2)$,

(ii) $m_{k}(g, H)=\mu(g, H)+o\left(k^{-1 / 2}\right)$ where $m_{k}(g, H):=\frac{1}{k} \sum_{i=1}^{k} g(i /(k+1)) H(i /(k+1))$,

(iii) $\sigma^{2}(g, H)$ converges absolutely,

(iv) $\int_{0}^{1} g(x) H^{\prime}(x)[x(1-x)]^{1 / 2} d x$ converges absolutely.

Then for $U_{1}, \ldots, U_{k}$ a sample from the standard uniform distribution, we have

$$
\sqrt{k}\left(\frac{\frac{1}{k} \sum_{i=1}^{k} g\left(\frac{i}{k+1}\right) H\left(U_{i, k}\right)-\mu(g, H)}{\sigma(g, H)}\right) \underset{k \rightarrow \infty}{\stackrel{d}{\longrightarrow}} N(0,1) .
$$

This result will be applied in the particular cases given by

Lemma 2. Let $g$ verifying Assumption $(\mathcal{G})$ and let $H$ stands for $I_{\alpha}, J_{\alpha, \rho}, K_{\alpha, \rho}$ or $L_{\alpha, \rho, \beta}$ with $\alpha>0, \rho<0$ and $\beta<0$ defined in Section 2.2. Then the functions $g$ and $H$ satisfy the hypotheses of Theorem 1.

Proof of Lemma 2. We prove the lemma when $H$ stands for $I_{\alpha}$. Similar arguments may be used in the other cases.

$\star$ Let $\tau_{0} \in(0,1)$ and $K>1$. In order to satisfy $M^{-1}<\frac{I_{\alpha}^{\prime}\left(u_{1}\right)}{I_{\alpha}^{\prime}\left(u_{2}\right)}<M$ whenever $0<u_{1}<\tau_{0}, 0<u_{2}<\tau_{0}$ and $K^{-1}<\frac{u_{1}}{u_{2}}<K$, it suffices to choose $M:=\max \left(K\left(1+\frac{\log K}{\log \tau_{0}^{-1}}\right)^{1-\alpha}, K\left(1+\frac{\log K}{\log \tau_{0}^{-1}}\right)^{\alpha-1}\right)$.

$\star$ One can check that for $M:=\max \left(\frac{1}{1-\tau_{0}}\left(\frac{K}{1-\tau_{0}}\right)^{\alpha-1}, \frac{1}{1-\tau_{0}}\left(\frac{K}{1-\tau_{0}}\right)^{1-\alpha}\right)$ we have $M^{-1}<\frac{I_{\alpha}^{\prime}\left(u_{1}\right)}{I_{\alpha}^{\prime}\left(u_{2}\right)}<M$ for 
$1-\tau_{0}<u_{1}<1,1-\tau_{0}<u_{2}<1$ and $K^{-1}<\frac{1-u_{1}}{1-u_{2}}<K$.

* Taking into account Assumption $(\mathcal{G}),(i)$ is obvious. Let us prove $(i i)$. From Assumption $(\mathcal{G})$, the function $x \mapsto g(x) I_{\alpha}(x)$ is positive, non increasing and integrable on $(0,1)$. We get easily the following inequalities

$$
\frac{1}{\sqrt{k}} \mu\left(g, I_{\alpha}\right)-\frac{k+1}{\sqrt{k}} \int_{0}^{1 /(k+1)} g(x) I_{\alpha}(x) d x \leq \sqrt{k}\left[m_{k}\left(g, I_{\alpha}\right)-\mu\left(g, I_{\alpha}\right)\right] \leq \frac{1}{\sqrt{k}} \mu\left(g, I_{\alpha}\right)-\frac{k+1}{\sqrt{k}} \int_{k /(k+1)}^{1} g(x) I_{\alpha}(x) d x .
$$

Since there exists $\delta>1 / 2$ such that $\int_{0}^{1} g(x) x^{-\delta} d x<\infty$ and $\int_{0}^{1} g(x)(1-x)^{-\delta} d x<\infty$, we see that

$$
\int_{0}^{1 /(k+1)} g(x) I_{\alpha}(x) d x=O\left((k+1)^{-\delta}(\log (k+1))^{\alpha}\right) \quad \text { and } \quad \int_{k /(k+1)}^{1} g(x) I_{\alpha}(x) d x=O\left((k+1)^{-\delta-\alpha}\right) .
$$

Now remark that the point $(i i i)$ is a direct consequence of $(i v)$ since

$$
\forall x, y \in(0,1) \quad x \wedge y(1-x \vee y) \leq(x(1-x))^{1 / 2}(y(1-y))^{1 / 2} .
$$

When $\alpha \geq 1$, we write

$$
\int_{0}^{1}\left|g(x) I_{\alpha}^{\prime}(x)(x(1-x))^{1 / 2}\right| d x=\alpha \int_{0}^{1} g(x) x^{-\delta} x^{\delta-1 / 2}\left(\log x^{-1}\right)^{\alpha-1}(1-x)^{1 / 2} d x .
$$

Then the point $(i v)$ follows from the boundedness of $x \mapsto x^{\delta-1 / 2}\left(\log x^{-1}\right)^{\alpha-1}$ and $x \mapsto(1-x)^{1 / 2}$. Similarly, when $\alpha \in(0,1)$ we use the boundedness of $x \mapsto(1-x)^{1 / 2}\left(\log x^{-1}\right)^{\alpha-1}$ and $x \mapsto x^{\delta-1 / 2}$.

\subsection{Proofs of Section 3}

Proof of Proposition 1. We recall that $\Gamma_{n, k}(g, \alpha)$ may be written as the quotient $M_{n, k}(g, \alpha) / \mu\left(g, I_{\alpha}\right)$. Since Assumption $(\mathcal{G})$ implies $\mu\left(g, I_{\alpha}\right) \neq 0$, we need to prove that $M_{n, k}(g, \alpha)={ }^{d} \gamma^{\alpha} \mu\left(g, I_{\alpha}\right)+o_{\mathbb{P}}(1)$.

Let $Y_{1}, \ldots, Y_{n}$ be independent and identically distributed with distribution $\left(1-y^{-1}\right), y>1$. Then we have $\left\{X_{n-k, n}, \ldots, X_{n, n}\right\}={ }^{d}\left\{U\left(Y_{n-k, n}\right), \ldots, U\left(Y_{n, n}\right)\right\}$ and consequently

$$
M_{n, k}(g, \alpha)={ }^{d} \frac{1}{k} \sum_{i=1}^{k} g\left(\frac{i}{k+1}\right) \log \left(\frac{U\left(Y_{n-i+1, n}\right)}{U\left(Y_{n-k, n}\right)}\right)^{\alpha} .
$$

Under Assumption $\left(\mathcal{H}_{1}\right)$, it can be proved as in Hüsler et al. (2006) that

$$
M_{n, k}(g, \alpha)={ }^{d}\left(\gamma^{\alpha}+o(1)\right) \frac{1}{k} \sum_{i=1}^{k} g\left(\frac{i}{k+1}\right)\left(\log \frac{Y_{n-i+1, n}}{Y_{n-k, n}}\right)^{\alpha}+o(1) \frac{1}{k} \sum_{i=1}^{k} g\left(\frac{i}{k+1}\right)
$$

Now $\left\{\log Y_{n-i+1, n}-\log Y_{n-k, n}\right\}_{i=1}^{k}=^{d}\left\{E_{n-i+1, n}-E_{n-k, n}\right\}_{i=1}^{k}=^{d}\left\{E_{k-i+1, k}^{\star}\right\}_{i=1}^{k}={ }^{d}\left\{\log U_{i, k}^{-1}\right\}_{i=1}^{k}$ where $E_{1}, \ldots, E_{n}$ and $E_{1}^{\star}, \ldots, E_{k}^{\star}$ are both samples from the standard exponential distribution, and $U_{1}, \ldots, U_{k}$ is a sample from the standard uniform distribution. Hence last expansion of $M_{n, k}(g, \alpha)$ becomes

$$
M_{n, k}(g, \alpha)={ }^{d}\left(\gamma^{\alpha}+o(1)\right) \frac{1}{k} \sum_{i=1}^{k} g\left(\frac{i}{k+1}\right)\left(\log U_{i, k}^{-1}\right)^{\alpha}+o(1) \frac{1}{k} \sum_{i=1}^{k} g\left(\frac{i}{k+1}\right) .
$$

From Assumption $(\mathcal{G})$, or more precisely using the fact that $m_{k}\left(g, I_{\alpha}\right)=\mu\left(g, I_{\alpha}\right)+o\left(k^{-1 / 2}\right)$, we obtain

$$
\frac{1}{k} \sum_{i=1}^{k} g\left(\frac{i}{k+1}\right)\left(\log U_{i, k}^{-1}\right)^{\alpha}=(1+o(1)) \mu\left(g, I_{\alpha}\right)+\frac{1}{k} \sum_{i=1}^{k} g\left(\frac{i}{k+1}\right)\left[\left(\log U_{i, k}^{-1}\right)^{\alpha}-\left(\log \frac{k+1}{i}\right)^{\alpha}\right]
$$

Let $c_{k}=k^{-1 / 2}$. We split the last term of (7) into two sums: when $i \in\left\{1, \ldots, k c_{k}\right\}$ and when $i \in\left\{k c_{k}+1, \ldots, k\right\}$. On the one hand, we find that on the set $\left\{U_{1, k} \geq k^{-2}\right\}$

$$
\left|\frac{1}{k} \sum_{i=1}^{k c_{k}} g\left(\frac{i}{k+1}\right)\left[\left(\log U_{i, k}^{-1}\right)^{\alpha}-\left(\log \frac{k+1}{i}\right)^{\alpha}\right]\right| \leq \frac{3^{\alpha}}{k} \sum_{i=1}^{k c_{k}} g\left(\frac{i}{k+1}\right)(\log (k+1))^{\alpha}
$$


By Assumption $(\mathcal{G})$, there exists $\delta=\delta(g)>1 / 2$ satisfying $\int_{0}^{1} g(x) x^{-\delta} d x<\infty$, so that

$$
\frac{1}{k} \sum_{i=1}^{k c_{k}} g\left(\frac{i}{k+1}\right) \sim \int_{0}^{c_{k}} g(x) d x=O\left(c_{k}^{\delta}\right)
$$

Since $\mathbb{P}\left(U_{1, k} \leq k^{-2}\right) \sim k^{-1}$ and $c_{k}^{\delta}(\log (k+1))^{\alpha}$ tends to 0 as $k$ tends to infinity, we proved

$$
\frac{1}{k} \sum_{i=1}^{k c_{k}} g\left(\frac{i}{k+1}\right)\left[\left(\log U_{i, k}^{-1}\right)^{\alpha}-\left(\log \frac{k+1}{i}\right)^{\alpha}\right] \stackrel{\mathbb{P}}{\rightarrow} 0
$$

On the other hand, from Lemma 3.2. of Hüsler et al. (2006) $\sup _{k c_{k}+1 \leq i \leq k}\left|\frac{k+1}{i} U_{i, k}-1\right|=o_{\mathbb{P}}(1)$, so that

$$
\frac{1}{k} \sum_{i=k c_{k}+1}^{k} g\left(\frac{i}{k+1}\right)\left[\left(\log U_{i, k}^{-1}\right)^{\alpha}-\left(\log \frac{k+1}{i}\right)^{\alpha}\right]=o_{\mathbb{P}}(1) \frac{1}{k} \sum_{i=k c_{k}+1}^{k} g\left(\frac{i}{k+1}\right)\left(\log \frac{k+1}{i}\right)^{\alpha-1} .
$$

Using Assumption $(\mathcal{G})$, we know that $\int_{0}^{1} g(x)(1-x)^{-\delta} d x<\infty$ and one may deduce that

$$
\frac{1}{k} \sum_{i=k c_{k}+1}^{k} g\left(\frac{i}{k+1}\right)\left[\left(\log U_{i, k}^{-1}\right)^{\alpha}-\left(\log \frac{k+1}{i}\right)^{\alpha}\right] \stackrel{\mathbb{P}}{\rightarrow} 0
$$

The result follows from the combination of (6), (7), (8) and (9).

Proof of Proposition 2. We use here the same notations as those introduced in the first lines of the proof of Proposition 1. Remark that $(k / n) Y_{n-k, n} \rightarrow 1$ in probability yields $A\left(Y_{n-k, n}\right)=A(n / k)\left(1+o_{\mathbb{P}}(1)\right)$. Recall also that $Y_{n-i+1, n} / Y_{n-k, n}$ is distributed as $U_{i, k}^{-1}$ for $U_{1}, \ldots, U_{k}$ a sample from the standard uniform distribution. As in Gomes and Martins (2001), it can be proved that under Assumption $\left(\mathcal{H}_{2}\right)$

$$
M_{n, k}(g, \alpha)={ }^{d} \gamma^{\alpha} T_{1, k}(g, \alpha)+A(n / k)\left(1+o_{\mathbb{P}}(1)\right) \alpha \gamma^{\alpha-1} T_{2, k}(g, \alpha, \rho)+o_{\mathbb{P}}(A(n / k)) \frac{1}{k} \sum_{i=1}^{k} g\left(\frac{i}{k+1}\right)
$$

with

$$
\begin{aligned}
T_{1, k}(g, \alpha) & :=\frac{1}{k} \sum_{i=1}^{k} g\left(\frac{i}{k+1}\right)\left(\log U_{i, k}^{-1}\right)^{\alpha} \\
T_{2, k}(g, \alpha, \rho) & :=\frac{1}{k} \sum_{i=1}^{k} g\left(\frac{i}{k+1}\right)\left(\log U_{i, k}^{-1}\right)^{\alpha-1} \frac{U_{i, k}^{-\rho}-1}{\rho} .
\end{aligned}
$$

Applying Theorem 1 to the function $I_{\alpha}$ we get

$$
T_{1, k}(g, \alpha)=\mu\left(g, I_{\alpha}\right)+\sigma\left(g, I_{\alpha}\right) \frac{P_{k}(g, \alpha)}{\sqrt{k}}
$$

where $P_{k}(g, \alpha)$ is asymptotically standard normal. The weak consistency contained in Theorem 1 for the function $J_{\alpha, \rho}$ yields

$$
T_{2, k}(g, \alpha, \rho) \stackrel{\mathbb{P}}{\rightarrow} \mu\left(g, J_{\alpha, \rho}\right)
$$

Combining what precedes we have

$$
M_{n, k}(g, \alpha)={ }^{d} \gamma^{\alpha}\left(\mu\left(g, I_{\alpha}\right)+\sigma\left(g, I_{\alpha}\right) \frac{P_{k}(g, \alpha)}{\sqrt{k}}\right)+A(n / k) \alpha \gamma^{\alpha-1} \mu\left(g, J_{\alpha, \rho}\right)+o_{\mathbb{P}}(A(n / k))
$$

since $\frac{1}{k} \sum_{i=1}^{k} g\left(\frac{i}{k+1}\right)=O(1)$ under Assumption $(\mathcal{G})$. The normalization by $\mu\left(g, I_{\alpha}\right)$ gives the result for $\Gamma_{n, k}(g, \alpha)$. 
Proof of Lemma 1. It is a direct consequence of Corollary 4 of Chernoff et al. (1967) which asserts that if the convergence in Theorem 1 applies for $g_{j}$ and $H_{j}$ with $j \in\{1, \ldots, r\}$, then it applies vectorially. Moreover, elements of the covariance matrix are computed from the formula

$$
\int_{0}^{1} \int_{0}^{1} g_{i}(x) H_{i}^{\prime}(x) g_{j}(y) H_{j}^{\prime}(y)(x \vee y)(1-x \wedge y) d x d y .
$$

Proof of Corollary 3. By definition of $x_{p}$ and $a_{n}$ we can write

$$
\log \hat{x}_{n, k, p}^{\Gamma}(g, \alpha)-\log x_{p}=\Gamma_{n, k}^{1 / \alpha}(g, \alpha) \log a_{n}+\log X_{n-k, n}-\log U\left(p^{-1}\right) .
$$

On the one hand, under Assumption $\left(\mathcal{H}_{2}\right)$, we know from Drees (1998) the following inequality: $\forall \varepsilon>0$, $\exists t_{0}=t_{0}(\varepsilon)$ such that $\forall t \geq t_{0}$ and $x \geq 1$,

$$
\left|\frac{\log U(t x)-\log U(t)-\gamma \log x}{A(t)}-\frac{x^{\rho}-1}{\rho}\right| \leq \varepsilon x^{\rho+\varepsilon} .
$$

Let $Y_{1}, \ldots, Y_{n}$ be a sample from the distribution function $\left(1-y^{-1}\right) 1_{y \geq 1}$. Recall that $X_{n-k, n}={ }^{d} U\left(Y_{n-k, n}\right)$. We apply this inequality to $t=n / k$ and $x=(k / n) Y_{n-k, n}$. We get for any $\varepsilon>0$

$$
\log X_{n-k, n}-\log U(n / k)={ }^{d} \gamma \log \left(\frac{k}{n} Y_{n-k, n}\right)+A(n / k) \frac{\left(\frac{k}{n} Y_{n-k, n}\right)^{\rho}-1}{\rho}+o(1) A(n / k)\left(\frac{k}{n} Y_{n-k, n}\right)^{\rho+\varepsilon} .
$$

Since $k$ is an intermediate number such that $\sqrt{k} A(n / k) \rightarrow \lambda$ and using the fact that

$$
\sqrt{k}\left(\frac{k}{n} Y_{n-k, n}-1\right) \stackrel{d}{\rightarrow} N(0,1)
$$

(see for instance de Haan and Ferreira, 2006, Corollary 2.2.2) we deduce easily that

$$
\frac{\sqrt{k}}{\log a_{n}}\left(\log X_{n-k, n}-\log U(n / k)\right)=o_{\mathbb{P}}(1) .
$$

Similarly, it can be proved that

$$
\frac{\sqrt{k}}{\log a_{n}}\left(\log U(n / k)-\log U\left(\frac{n+1}{k+1}\right)\right)=o(1) .
$$

On the other hand, we have for any $\varepsilon>0$

$$
\log U\left(\frac{n+1}{k+1}\right)-\log U\left(p^{-1}\right)=-\gamma \log a_{n}-A\left(\frac{n+1}{k+1}\right) \frac{a_{n}^{\rho}-1}{\rho}+o(1) A\left(\frac{n+1}{k+1}\right) a_{n}^{\rho+\varepsilon} .
$$

Since it is assumed that $\rho<0$ one can choose $\varepsilon>0$ such that $\rho+\varepsilon<0$. Then, using the fact that $\sqrt{k} A((n+1) /(k+1)) \rightarrow \lambda$, we obtain

$$
\frac{\sqrt{k}}{\log a_{n}}\left(\log U\left(\frac{n+1}{k+1}\right)-\log U\left(p^{-1}\right)+\gamma \log a_{n}\right)=o(1) .
$$

In summary, (10) combined with (11), (12) and (13) yields

$$
\frac{\sqrt{k}}{\log a_{n}}\left(\log \hat{x}_{n, k, p}^{\Gamma}(g, \alpha)-\log x_{p}\right)={ }^{d} \sqrt{k}\left(\Gamma_{n, k}^{1 / \alpha}(g, \alpha)-\gamma\right)+o_{\mathbb{P}}(1) .
$$

The result follows by application of Corollary 1 . 


\subsection{Proofs of Section 4}

Proof of Proposition 3. Using the same arguments as in Proposition 3.2 of Gomes et al. (2002), we may write that

$$
\begin{gathered}
M_{n, k}(g, \alpha)={ }^{d \quad} \gamma^{\alpha} T_{1, k}(g, \alpha)+A(n / k) \alpha \gamma^{\alpha-1} T_{2, k}(g, \alpha, \rho)+A^{2}(n / k)\left(1+o_{\mathbb{P}}(1)\right) \frac{\alpha(\alpha-1)}{2} \gamma^{\alpha-2} T_{3, k}(g, \alpha, \rho) \\
+A(n / k) B(n / k)(1+o \mathbb{P}(1)) \alpha \gamma^{\alpha-1} T_{4, k}(g, \alpha, \rho, \beta)
\end{gathered}
$$

with $T_{1, k}(g, \alpha)$ and $T_{2, k}(g, \alpha, \rho)$ already defined in the proof of Proposition 2 and where

$$
\begin{aligned}
T_{3, k}(g, \alpha, \rho) & :=\frac{1}{k} \sum_{i=1}^{k} g\left(\frac{i}{k+1}\right)\left(\log U_{i, k}^{-1}\right)^{\alpha-2}\left(\frac{U_{i, k}^{-\rho}-1}{\rho}\right)^{2}, \\
T_{4, k}(g, \alpha, \rho, \beta) & :=\frac{1}{k} \sum_{i=1}^{k} g\left(\frac{i}{k+1}\right)\left(\log U_{i, k}^{-1}\right)^{\alpha-1} \frac{1}{\beta}\left(\frac{U_{i, k}^{-(\rho+\beta)}-1}{\rho+\beta}-\frac{U_{i, k}^{-\rho}-1}{\rho}\right) .
\end{aligned}
$$

By application of Theorem 1 to the function $J_{\alpha, \rho}$, we get

$$
T_{2, k}(g, \alpha, \rho)=\mu\left(g, J_{\alpha, \rho}\right)+\sigma\left(g, J_{\alpha, \rho}\right) \frac{\bar{P}_{k}(g, \alpha, \rho)}{\sqrt{k}}
$$

where $\bar{P}_{k}(g, \alpha, \rho)$ is asymptotically standard normal. Now, we apply the weak consistency, contained in Theorem 1 , to the functions $K_{\alpha, \rho}$ and $L_{\alpha, \rho, \beta}$ in order to get

$$
\begin{array}{r}
T_{3, k}(g, \alpha, \rho) \stackrel{\mathbb{P}}{\rightarrow} \mu\left(g, K_{\alpha, \rho}\right), \\
T_{4, k}(g, \alpha, \rho, \beta) \stackrel{\mathbb{P}}{\rightarrow} \mu\left(g, L_{\alpha, \rho, \beta}\right) .
\end{array}
$$

The relations (14), (15), (16), (17) lead to the conclusion.

Proof of Proposition 4. Let $g_{1}$ and $g_{2}$ be some weighted functions satisfying $(\mathcal{G})$. When $\left(\mathcal{H}_{2}\right)$ and $(\mathcal{K})$ hold we can expand

$$
\begin{aligned}
\Gamma_{n, k}\left(g_{1}, \alpha\right)-\Gamma_{n, k}\left(g_{2}, 1\right)^{\alpha}={ }^{d} & \frac{\gamma^{\alpha}}{\sqrt{k}}\left(\bar{\sigma}\left(g_{1}, I_{\alpha}\right) P_{k}\left(g_{1}, \alpha\right)-\alpha \bar{\sigma}\left(g_{2}, I_{1}\right) P_{k}\left(g_{2}, 1\right)\right) \\
& +\alpha \gamma^{\alpha-1}\left(\bar{\mu}\left(g_{1}, J_{\alpha, \rho}\right)-\bar{\mu}\left(g_{2}, J_{1, \rho}\right)\right) A(n / k)\left(1+o_{\mathbb{P}}(1)\right) .
\end{aligned}
$$

For sequences $k=k(n)$ such that $\sqrt{k} A(n / k)$ tends to infinity as $n \rightarrow \infty$, this implies

$$
\frac{\Gamma_{n, k}\left(g_{1}, \alpha\right)-\Gamma_{n, k}\left(g_{2}, 1\right)^{\alpha}}{A(n / k)} \stackrel{\mathbb{P}}{\rightarrow} \alpha \gamma^{\alpha-1}\left(\bar{\mu}\left(g_{1}, J_{\alpha, \rho}\right)-\bar{\mu}\left(g_{2}, J_{1, \rho}\right)\right) .
$$

Under Assumption $(\mathcal{G})$ and if $\psi\left(\boldsymbol{g}, \alpha_{1}, \alpha_{2}, \rho\right)$ and $\psi\left(\boldsymbol{h}, \alpha_{3}, \alpha_{4}, \rho\right)$ are well defined and nonzero, it follows that

$$
\Psi_{n, k}\left(\boldsymbol{g}, \alpha_{1}, \alpha_{2}\right) \stackrel{\mathbb{P}}{\rightarrow} \gamma^{\alpha_{1}-\alpha_{2}} \psi\left(\boldsymbol{g}, \alpha_{1}, \alpha_{2}, \rho\right)
$$

and

$$
\frac{\Psi_{n, k}\left(\boldsymbol{g}, \alpha_{1}, \alpha_{2}\right)}{\left[\Psi_{n, k}\left(\boldsymbol{h}, \alpha_{3}, \alpha_{4}\right)\right]^{2}} \stackrel{\mathbb{P}}{\rightarrow} \gamma^{\alpha_{1}-\alpha_{2}-\left(\alpha_{3}-\alpha_{4}\right)} \frac{\psi\left(\boldsymbol{g}, \alpha_{1}, \alpha_{2}, \rho\right)}{\psi^{2}\left(\boldsymbol{h}, \alpha_{3}, \alpha_{4}, \rho\right)} .
$$

The rest is straightforward taking into account the values of the $\alpha_{i}^{\prime} s$ in Proposition 4 .

Proof of Proposition 5. If $g_{1}$ and $g_{2}$ satisfy $(\mathcal{G})$, if $\left(\mathcal{H}_{3}\right)$ and $(\mathcal{K})$ hold we have

$$
\begin{aligned}
\Gamma_{n, k}\left(g_{1}, \alpha\right)-\Gamma_{n, k}\left(g_{2}, 1\right)^{\alpha}={ }^{d} & \frac{\gamma^{\alpha}}{\sqrt{k}}\left(\bar{\sigma}\left(g_{1}, I_{\alpha}\right) P_{k}\left(g_{1}, \alpha\right)-\alpha \bar{\sigma}\left(g_{2}, I_{1}\right) P_{k}\left(g_{2}, 1\right)\right) \\
& +\alpha \gamma^{\alpha-1}\left(\bar{\mu}\left(g_{1}, J_{\alpha, \rho}\right)-\bar{\mu}\left(g_{2}, J_{1, \rho}\right)\right) A(n / k) \\
& +\alpha \gamma^{\alpha-1}\left(\bar{\sigma}\left(g_{1}, J_{\alpha, \rho}\right) \bar{P}_{k}\left(g_{1}, \alpha, \rho\right)-\bar{\sigma}\left(g_{2}, J_{1, \rho}\right) \bar{P}_{k}\left(g_{2}, 1, \rho\right)\right) \frac{A(n / k)}{\sqrt{k}} \\
& +\frac{\alpha(\alpha-1)}{2} \gamma^{\alpha-2}\left(\bar{\mu}\left(g_{1}, K_{\alpha, \rho}\right)-\bar{\mu}^{2}\left(g_{2}, J_{1, \rho}\right)\right) A^{2}(n / k)\left(1+o_{\mathbb{P}}(1)\right) \\
& +\alpha \gamma^{\alpha-1}\left(\bar{\mu}\left(g_{1}, L_{\alpha, \rho, \beta}\right)-\bar{\mu}\left(g_{2}, L_{1, \rho, \beta}\right)\right) A(n / k) B(n / k)\left(1+o_{\mathbb{P}}(1)\right) .
\end{aligned}
$$


Moreover for $k=k(n)$ such that $\sqrt{k} A(n / k)$ tends to infinity as $n \rightarrow \infty$ we see easily that

$$
\begin{aligned}
\frac{\Gamma_{n, k}\left(g_{1}, \alpha\right)-\Gamma_{n, k}\left(g_{2}, 1\right)^{\alpha}}{A(n / k)}=^{d} & \alpha \gamma^{\alpha-1}\left(\bar{\mu}\left(g_{1}, J_{\alpha, \rho}\right)-\bar{\mu}\left(g_{2}, J_{1, \rho}\right)\right) \\
& +\frac{\gamma^{\alpha}}{\sqrt{k} A(n / k)}\left(\bar{\sigma}\left(g_{1}, I_{\alpha}\right) P_{k}\left(g_{1}, \alpha\right)-\alpha \bar{\sigma}\left(g_{2}, I_{1}\right) P_{k}\left(g_{2}, 1\right)\right) \\
& +\frac{\alpha(\alpha-1)}{2} \gamma^{\alpha-2}\left(\bar{\mu}\left(g_{1}, K_{\alpha, \rho}\right)-\bar{\mu}^{2}\left(g_{2}, J_{1, \rho}\right)\right) A(n / k)\left(1+o_{\mathbb{P}}(1)\right) \\
& +\alpha \gamma^{\alpha-1}\left(\bar{\mu}\left(g_{1}, L_{\alpha, \rho, \beta}\right)-\bar{\mu}\left(g_{2}, L_{1, \rho, \beta}\right)\right) B(n / k)\left(1+o_{\mathbb{P}}(1)\right) .
\end{aligned}
$$

Whenever the weighted functions satisfy $(\mathcal{G})$ and are such that $\bar{\mu}\left(g_{2}, J_{\alpha_{2}, \rho}\right) \neq \bar{\mu}\left(g_{4}, J_{1, \rho}\right)$, it follows that

$$
\begin{array}{r}
\Psi_{n, k}\left(\boldsymbol{g}, \alpha_{1}, \alpha_{2}\right)={ }^{d} \gamma^{\alpha_{1}-\alpha_{2}} \psi\left(\boldsymbol{g}, \alpha_{1}, \alpha_{2}, \rho\right)+\frac{\gamma^{\alpha_{1}-\alpha_{2}+1}}{\sqrt{k} A(n / k)}\left(\frac{\alpha_{2} V_{k}\left(g_{1}, g_{3}, \alpha_{1}\right)-\alpha_{1} V_{k}\left(g_{2}, g_{4}, \alpha_{2}\right) \psi\left(\boldsymbol{g}, \alpha_{1}, \alpha_{2}, \rho\right)}{\alpha_{1} \alpha_{2}\left(\bar{\mu}\left(g_{2}, J_{\alpha_{2}, \rho}\right)-\bar{\mu}\left(g_{4}, J_{1, \rho}\right)\right)}\right) \\
+\frac{1}{2} \gamma^{\alpha_{1}-\alpha_{2}-1}\left(\frac{\left(\alpha_{1}-1\right)\left[\bar{\mu}\left(g_{1}, K_{\alpha_{1}, \rho}\right)-\bar{\mu}^{2}\left(g_{3}, J_{1, \rho}\right)\right]-\left(\alpha_{2}-1\right)\left[\bar{\mu}\left(g_{2}, K_{\alpha_{2}, \rho}\right)-\bar{\mu}^{2}\left(g_{4}, J_{1, \rho}\right)\right] \psi\left(\boldsymbol{g}, \alpha_{1}, \alpha_{2}, \rho\right)}{\bar{\mu}\left(g_{2}, J_{\alpha_{2}, \rho}\right)-\bar{\mu}\left(g_{4}, J_{1, \rho}\right)}\right) A(n / k)(1+o \mathbb{P}(1)) \\
+\gamma^{\alpha_{1}-\alpha_{2}}\left(\frac{\left[\bar{\mu}\left(g_{1}, L_{\alpha_{1}, \rho, \beta}\right)-\bar{\mu}\left(g_{3}, L_{1, \rho, \beta}\right)\right]-\left[\bar{\mu}\left(g_{2}, L_{\alpha_{2}, \rho, \beta}\right)-\bar{\mu}\left(g_{4}, L_{1, \rho, \beta}\right)\right] \psi\left(\boldsymbol{g}, \alpha_{1}, \alpha_{2}, \rho\right)}{\bar{\mu}\left(g_{2}, J_{\alpha_{2}, \rho}\right)-\bar{\mu}\left(g_{4}, J_{1, \rho}\right)}\right) B(n / k)\left(1+o_{\mathbb{P}}(1)\right)
\end{array}
$$

where $V_{k}(g, h, \alpha):=\bar{\sigma}\left(g, I_{\alpha}\right) P_{k}(g, \alpha)-\alpha \bar{\sigma}\left(h, I_{1}\right) P_{k}(h, 1)$.

Let us introduce the process $W_{k}\left(\boldsymbol{g}, \alpha_{1}, \alpha_{2}, \rho\right):=\frac{\alpha_{2} V_{k}\left(g_{1}, g_{3}, \alpha_{1}\right)-\alpha_{1} V_{k}\left(g_{2}, g_{4}, \alpha_{2}\right) \psi\left(\boldsymbol{g}, \alpha_{1}, \alpha_{2}, \rho\right)}{\alpha_{1} \alpha_{2}\left(\bar{\mu}\left(g_{2}, J_{\alpha_{2}, \rho}\right)-\bar{\mu}\left(g_{4}, J_{1, \rho}\right)\right) \psi\left(\boldsymbol{g}, \alpha_{1}, \alpha_{2}, \rho\right)}$. Thus, under the assumptions of Proposition 5 and using $(1+x)^{2}=1+2 x+o(x)$ and $1 /(1+x)=1-x+o(x)$ as $x \rightarrow 0$, we get

$$
\begin{array}{rl}
S_{n, k}(\boldsymbol{g}, \boldsymbol{h}, \alpha, \ell)=^{d} & s(\boldsymbol{g}, \boldsymbol{h}, \alpha, \ell, \rho)\left[1+\gamma \frac{W_{k}(\boldsymbol{g}, 2 \alpha, 2 \ell, \rho)-2 W_{k}(\boldsymbol{h}, \alpha+\ell, 2 \ell, \rho)}{\sqrt{k} A(n / k)}\right. \\
& +\frac{m_{A}^{S}(\boldsymbol{g}, 2 \alpha, 2 \ell, \rho)-2 m_{A}^{S}(\boldsymbol{h}, \alpha+\ell, 2 \ell, \rho)}{2 \gamma} A(n / k) \\
& \left.+\left(m_{B}^{S}(\boldsymbol{g}, 2 \alpha, 2 \ell, \rho, \beta)-2 m_{B}^{S}(\boldsymbol{h}, \alpha+\ell, 2 \ell, \rho, \beta)\right) B(n / k)\right]\left(1+o_{\mathbb{P}}(1)\right)
\end{array}
$$

with $m_{A}^{S}$ and $m_{B}^{S}$ introduced in Section 4.1. Then we can conclude since $\kappa_{S}\left(g_{1}, g_{3}, h_{1}, h_{3}, \alpha_{1}, \alpha_{3}\right)$ is the asymptotic covariance between $V_{k}\left(g_{1}, g_{3}, \alpha_{1}\right)$ and $V_{k}\left(h_{1}, h_{3}, \alpha_{3}\right)$ and since $c_{S}\left(\boldsymbol{g}, \boldsymbol{h}, \alpha_{1}, \alpha_{2}, \alpha_{3}, \alpha_{4}, \rho\right)$ is the asymptotic covariance between $W_{k}\left(\boldsymbol{g}, \alpha_{1}, \alpha_{2}, \rho\right)$ and $W_{k}\left(\boldsymbol{h}, \alpha_{3}, \alpha_{4}, \rho\right)$.

Proof of Proposition 6. The proof is a direct consequence of the convergence obtained in (18).

Proof of Proposition 7. With the same arguments as those used in the proof of Proposition 5, one checks that

$$
\begin{array}{r}
Q_{n, k}\left(\boldsymbol{g}, \boldsymbol{h}, \alpha_{g}, \alpha_{h}, \ell\right)={ }^{d} q\left(\boldsymbol{g}, \boldsymbol{h}, \alpha_{g}, \alpha_{h}, \ell, \rho\right)\left[1+\gamma \frac{W_{k}\left(\boldsymbol{g}, \alpha_{g}, \alpha_{g}+\ell, \rho\right)-W_{k}\left(\boldsymbol{h}, \alpha_{h}, \alpha_{h}+\ell, \rho\right)}{\sqrt{k} A(n / k)}\right. \\
+\frac{m_{A}^{S}\left(\boldsymbol{g}, \alpha_{g}, \alpha_{g}+\ell, \rho\right)-m_{A}^{S}\left(\boldsymbol{h}, \alpha_{h}, \alpha_{h}+\ell, \rho\right)}{2 \gamma} A(n / k) \\
\left.+\left(m_{B}^{S}\left(\boldsymbol{g}, \alpha_{g}, \alpha_{g}+\ell, \rho, \beta\right)-m_{B}^{S}\left(\boldsymbol{h}, \alpha_{h}, \alpha_{h}+\ell, \rho, \beta\right)\right) B(n / k)\right]\left(1+o_{\mathbb{P}}(1)\right) .
\end{array}
$$

The result follows taking into account the covariances given at the end of the proof of Proposition 5 .

Proof of Proposition 8. First note that we may write

$$
\Phi_{n, k}(\boldsymbol{g}, \alpha, \boldsymbol{\theta}, \tau)=\frac{\left(\left(\frac{\Gamma_{n, k}\left(g_{1}, \alpha \theta_{1}\right)}{\gamma^{\alpha \theta_{1}}}\right)^{\tau / \theta_{1}}-\left(\frac{\Gamma_{n, k}\left(g_{2}, \alpha \theta_{2}\right)}{\gamma^{\alpha \theta_{2}}}\right)^{\tau / \theta_{2}}\right) / A(n / k)}{\left(\left(\frac{\Gamma_{n, k}\left(g_{3}, \alpha \theta_{3}\right)}{\gamma^{\alpha \theta_{3}}}\right)^{\tau / \theta_{3}}-\left(\frac{\Gamma_{n, k}\left(g_{4}, \alpha \theta_{4}\right)}{\gamma^{\alpha \theta_{4}}}\right)^{\tau / \theta_{4}}\right) / A(n / k)} .
$$

Using the distributional representation (3), we observe that under $\left(\mathcal{H}_{2}\right)$

$$
\left(\frac{\Gamma_{n, k}(g, \alpha \theta)}{\gamma^{\alpha \theta}}\right)^{\tau / \theta}={ }^{d} 1+\frac{\tau}{\theta} \bar{\sigma}\left(g, I_{\alpha \theta}\right) \frac{P_{k}(g, \alpha \theta)}{\sqrt{k}}+A(n / k)\left(1+o_{\mathbb{P}}(1)\right) \frac{\alpha \tau}{\gamma} \bar{\mu}\left(g, J_{\alpha \theta, \rho}\right)
$$


For sequences $k=k(n)$ such that $\sqrt{k} A(n / k)$ tends to infinity as $n \rightarrow \infty$ we have

$$
\frac{\left(\frac{\Gamma_{n, k}\left(g_{1} \alpha \theta_{1}\right)}{\gamma^{\alpha \theta_{1}}}\right)^{\tau / \theta_{1}}-\left(\frac{\Gamma_{n, k}\left(g_{2}, \alpha \theta_{2}\right)}{\gamma^{\alpha \theta_{2}}}\right)^{\tau / \theta_{2}}}{A(n / k)} \stackrel{\mathbb{P}}{\rightarrow} \frac{\alpha \tau}{\gamma}\left(\bar{\mu}\left(g_{1}, J_{\alpha \theta_{1}, \rho}\right)-\bar{\mu}\left(g_{2}, J_{\alpha \theta_{2}, \rho}\right)\right)
$$

and the result follows immediately.

Proof of Proposition 9. The distributional representation (5) implies

$$
\begin{aligned}
\left(\frac{\Gamma_{n, k}(g, \alpha \theta)}{\gamma^{\alpha \theta}}\right)^{\tau / \theta} & ={ }^{d} 1+\frac{\tau}{\theta} \bar{\sigma}\left(g, I_{\alpha \theta}\right) \frac{P_{k}(g, \alpha \theta)}{\sqrt{k}}+A(n / k) \frac{\alpha \tau}{\gamma} \bar{\mu}\left(g, J_{\alpha \theta, \rho}\right) \\
& +A(n / k) \frac{\alpha \tau}{\gamma} \bar{\sigma}\left(g, J_{\alpha \theta, \rho}\right) \frac{\bar{P}_{k}(g, \alpha \theta, \rho)}{\sqrt{k}}+A(n / k) B(n / k) \frac{\alpha \tau}{\gamma} \bar{\mu}\left(g, L_{\alpha \theta, \rho, \beta}\right)\left(1+o_{\mathbb{P}}(1)\right) \\
& +A^{2}(n / k) \frac{\alpha \tau}{2 \gamma^{2}}\left((\alpha \theta-1) \bar{\mu}\left(g, K_{\alpha \theta, \rho}\right)+\alpha(\tau-\theta) \bar{\mu}^{2}\left(g, J_{\alpha \theta, \rho}\right)\right)\left(1+o_{\mathbb{P}}(1)\right)
\end{aligned}
$$

Hence the numerator in the fraction (19) satisfies

$$
\begin{gathered}
\frac{\left(\frac{\Gamma_{n, k}\left(g_{1} \alpha \theta_{1}\right)}{\gamma^{\alpha \theta_{1}}}\right)^{\tau / \theta_{1}}-\left(\frac{\Gamma_{n, k}\left(g_{2}, \alpha \theta_{2}\right)}{\gamma^{\alpha \theta_{2}}}\right)^{\tau / \theta_{2}}}{A(n / k)}={ }^{d} \frac{\alpha \tau}{\gamma}\left(\bar{\mu}\left(g_{1}, J_{\alpha \theta_{1}, \rho}\right)-\bar{\mu}\left(g_{2}, J_{\alpha \theta_{2}, \rho}\right)\right)+\alpha \tau \frac{Z_{k}\left(g_{1}, g_{2}, \alpha \theta_{1}, \alpha \theta_{2}\right)}{A(n / k) \sqrt{k}} \\
+B(n / k)\left(1+o_{\mathbb{P}}(1)\right) \frac{\alpha \tau}{\gamma}\left(\bar{\mu}\left(g_{1}, L_{\alpha \theta_{1}, \rho, \beta}\right)-\bar{\mu}\left(g_{2}, L_{\alpha \theta_{2}, \rho, \beta}\right)\right)+A(n / k)\left(1+o_{\mathbb{P}}(1)\right) \frac{\alpha \tau}{2 \gamma^{2}} \times \\
\left(\left(\alpha \theta_{1}-1\right) \bar{\mu}\left(g_{1}, K_{\alpha \theta_{1}, \rho}\right)+\alpha\left(\tau-\theta_{1}\right) \bar{\mu}^{2}\left(g_{1}, J_{\alpha \theta_{1}, \rho}\right)-\left(\alpha \theta_{2}-1\right) \bar{\mu}\left(g_{2}, K_{\alpha \theta_{2}, \rho}\right)-\alpha\left(\tau-\theta_{2}\right) \bar{\mu}^{2}\left(g_{2}, J_{\alpha \theta_{2}, \rho}\right)\right)
\end{gathered}
$$

with $Z_{k}\left(g_{1}, g_{2}, \alpha_{1}, \alpha_{2}\right):=\frac{\bar{\sigma}\left(g_{1}, I_{\alpha_{1}}\right)}{\alpha_{1}} P_{k}\left(g_{1}, \alpha_{1}\right)-\frac{\bar{\sigma}\left(g_{2}, I_{\alpha_{2}}\right)}{\alpha_{2}} P_{k}\left(g_{2}, \alpha_{2}\right)$. Using the notations introduced in Section 4.3 , it yields

$$
\begin{array}{r}
\Phi_{n, k}(\boldsymbol{g}, \alpha, \boldsymbol{\theta}, \tau)=\phi(\boldsymbol{g}, \alpha, \boldsymbol{\theta}, \rho)+\frac{\gamma}{A(n / k) \sqrt{k}} \frac{Z_{k}\left(g_{1}, g_{2}, \alpha \theta_{1}, \alpha \theta_{2}\right)-Z_{k}\left(g_{3}, g_{4}, \alpha \theta_{3}, \alpha \theta_{4}\right) \phi(\boldsymbol{g}, \alpha, \boldsymbol{\theta}, \rho)}{\bar{\mu}\left(g_{3}, J_{\alpha \theta_{3}, \rho}\right)-\bar{\mu}\left(g_{4}, J_{\alpha \theta_{4}, \rho}\right)} \\
+B(n / k)\left(1+o_{\mathbb{P}}(1)\right) \frac{m_{\Phi}^{B}\left(g_{1}, g_{2}, \alpha \theta_{1}, \alpha \theta_{2}, \rho, \beta\right)-m_{\Phi}^{B}\left(g_{3}, g_{4}, \alpha \theta_{1}, \alpha \theta_{2}, \rho, \beta\right) \phi(\boldsymbol{g}, \alpha, \boldsymbol{\theta}, \rho)}{\bar{\mu}\left(g_{3}, J_{\alpha \theta_{3}, \rho}\right)-\bar{\mu}\left(g_{4}, J_{\alpha \theta_{4}, \rho}\right)} \\
+A(n / k)\left(1+o_{\mathbb{P}}(1)\right) \frac{m_{\Phi}^{A}\left(g_{1}, g_{2}, \alpha \theta_{1}, \alpha \theta_{2}, \alpha \tau, \rho\right)-m_{\Phi}^{A}\left(g_{3}, g_{4}, \alpha \theta_{3}, \alpha \theta_{4}, \alpha \tau, \rho\right) \phi(\boldsymbol{g}, \alpha, \boldsymbol{\theta}, \rho)}{2 \gamma\left(\bar{\mu}\left(g_{3}, J_{\alpha \theta_{3}, \rho}\right)-\bar{\mu}\left(g_{4}, J_{\alpha \theta_{4}, \rho}\right)\right)} .
\end{array}
$$

The result follows since the asymptotic covariance between $Z_{k}\left(g_{1}, g_{2}, \alpha_{1}, \alpha_{2}\right)$ and $Z_{k}\left(g_{3}, g_{4}, \alpha_{3}, \alpha_{4}\right)$ is given by $c_{\Phi}\left(g_{1}, g_{2}, g_{3}, g_{4}, \alpha_{1}, \alpha_{2}, \alpha_{3}, \alpha_{4}\right)$.

\section{References}

Beirlant, J., Vynckier, P., and Teugels, J. L. (1996). Excess functions and estimation of the extreme-value index. Bernoulli, 2(4):293-318.

Chernoff, H., Gastwirth, J. L., and Johns, Jr., M. V. (1967). Asymptotic distribution of linear combinations of functions of order statistics with applications to estimation. Ann. Math. Statist, 38:52-72.

Csörgő, S., Deheuvels, P., and Mason, D. (1985). Kernel estimates of the tail index of a distribution. Ann. Statist., 13(3):1050-1077.

de Haan, L. and Ferreira, A. (2006). Extreme value theory. Springer Series in Operations Research and Financial Engineering. Springer, New York. An introduction.

Dekkers, A. L. M. and de Haan, L. (1993). Optimal choice of sample fraction in extreme-value estimation. $J$. Multivariate Anal., 47(2):173-195.

Draisma, G., de Haan, L., Peng, L., and Pereira, T. T. (1999). A bootstrap-based method to achieve optimality in estimating the extreme-value index. Extremes, 2(4):367-404 (2000). 
Drees, H. (1998). On smooth statistical tail functionals. Scand. J. Statist., 25(1):187-210.

Drees, H. and Kaufmann, E. (1998). Selecting the optimal sample fraction in univariate extreme value estimation. Stochastic Process. Appl., 75(2):149-172.

Fils, A. and Guillou, A. (2004). A new extreme quantile estimator for heavy-tailed distributions. C. R. Math. Acad. Sci. Paris, 338(6):493-498.

Fraga Alves, M. I., Gomes, M. I., and de Haan, L. (2003). A new class of semi-parametric estimators of the second order parameter. Port. Math. (N.S.), 60(2):193-213.

Gomes, M. I., de Haan, L., and Peng, L. (2002). Semi-parametric estimation of the second order parameter in statistics of extremes. Extremes, 5(4):387-414 (2003).

Gomes, M. I. and Martins, M. J. (1999). Efficient alternatives to the hill estimator. Proceedings of the Workshop V.E.L.A. -Extreme Values and Additive Laws, C.E.A.U.L. editions-, pages 40-43.

Gomes, M. I. and Martins, M. J. (2001). Generalizations of the Hill estimator-asymptotic versus finite sample behaviour. J. Statist. Plann. Inference, 93(1-2):161-180.

Hall, P. (1982). On some simple estimates of an exponent of regular variation. Journal of the Royal Statistical Society. Series B (Methodological), 44(1):37-42.

Hall, P. and Welsh, A. H. (1985). Adaptive estimates of parameters of regular variation. Ann. Statist., 13(1):331-341.

Hill, B. M. (1975). A simple general approach to inference about the tail of a distribution. Ann. Statist., $3(5): 1163-1174$.

Hüsler, J., Li, D., and Müller, S. (2006). Weighted least squares estimation of the extreme value index. Statist. Probab. Lett., 76(9):920-930.

Kratz, M. and Resnick, S. I. (1996). The QQ-estimator and heavy tails. Comm. Statist. Stochastic Models, 12(4):699-724.

Schultze, J. and Steinebach, J. (1996). On least squares estimates of an exponential tail coefficient. Statist. Decisions, 14(4):353-372.

Segers, J. (2001). Residual estimators. J. Statist. Plann. Inference, 98(1-2):15-27.

Weissman, I. (1978). Estimation of parameters and large quantiles based on the $k$ largest observations. $J$. Amer. Statist. Assoc., 73(364):812-815. 